INSTITUT NATIONAL DE LA STATISTIQUE ET DES ETUDES ECONOMIQUES

Série des Documents de Travail du CREST

(Centre de Recherche en Economie et Statistique)

\author{
$\mathrm{n}^{\circ}$ 2009-18 \\ Combining Nonparametric \\ and Optimal Linear Time Series \\ Predictions \\ S. DABO-NIANG ${ }^{1}$ \\ C. FRANCQ ${ }^{2}$ \\ J.-M. ZAKOIAN ${ }^{3}$
}

Les documents de travail ne reflètent pas la position de l'INSEE et n'engagent que leurs auteurs.

Working papers do not reflect the position of INSEE but only the views of the authors.

\footnotetext{
1 Université Lille III, GREMARS-EQUIPPE, BP 60149, 59653 Villeneuve d'Ascq Cedex, France. Email : sophie.dabo@univ-lille3.fr

2 Université Lille III, GREMARS-EQUIPPE, BP 60149, 59653 Villeneuve d'Ascq Cedex, France. Email : christian.francq@univ.lille3.fr

3 CREST and EQUIPPE-GREMARS, 15 boulevard Gabriel Péri, 92245 Malakoff Cedex, France. Email : zakoian@ensae.fr
} 


\title{
Combining nonparametric and optimal linear time series predictions
}

\author{
Sophie Dabo-Niang, Christian Francq ${ }^{\dagger}$ and Jean-Michel Zakoïan ${ }^{\ddagger}$
}

\begin{abstract}
We introduce a semiparametric procedure for more efficient prediction of a strictly stationary process admitting an ARMA representation. The procedure is based on the estimation of the ARMA representation, followed by a nonparametric regression where the ARMA residuals are used as explanatory variables. Compared to standard nonparametric regression methods, the number of explanatory variables can be reduced because our approach exploits the linear dependence of the process. We establish consistency and asymptotic normality results. A Monte Carlo study and an empirical application on stock indices suggest that significant gains can be achieved with our approach.
\end{abstract}

Résumé: Nous introduisons une procédure semi-paramétrique afin de prévoir plus efficacement un processus strictement stationnaire admettant une représentation ARMA. Cette procédure est fondée sur l'estimation de la représentation ARMA, suivie d'une régression non paramétrique dans laquelle les résidus ARMA sont utilisés comme variables explicatives. Par rapport aux méthodes standard de régression non paramétrique, cette approche permet de réduire le nombre de variables explicatives car elle exploite la dépendance linéaire du processus. On établit des résultats de convergence et de normalité asymptotique. Une étude par simulation et une application sur données d'indices boursiers montrent que des gains d'efficacité significatifs peuvent être obtenus par cette méthode.

\footnotetext{
*Université Lille III, EQUIPPE-GREMARS, BP 60 149, 59653 Villeneuve d'Ascq cedex, France. E-mail: sophie.dabo@univ-lille3.fr

${ }^{\dagger}$ Université Lille III, EQUIPPE-GREMARS, BP 60 149, 59653 Villeneuve d'Ascq cedex, France. E-mail: christian.francq@univ-lille3.fr

${ }^{\ddagger}$ CREST and EQUIPPE-GREMARS, 15 Bd G. Péri, 92245 Malakoff Cedex, France. E-mail: zakoian@ensae.fr
} 


\section{Introduction}

After three decades of non-linear time series models, the class of ARMA models remains the most widely employed parametric family. Reasons can be found in the generality of the class (when the noise is only assumed to be uncorrelated), the relative ease of implementation and the ability to provide optimal linear predictions. If a stationary processes $\left(W_{t}\right)$ is an ARMA process, its optimal linear prediction

$$
E L\left(W_{t} \mid\left\{W_{u}, u<t\right\}\right)=\sum_{i=1}^{\infty} a_{i} W_{t-i},
$$

is obtained from the ARMA model. However, ARMA models also have important drawbacks. Their generality vanishes when strong assumptions (such as independence, martingale difference) are made on the noise. The optimal linear prediction does not coincide, in this case, with the optimal prediction

$$
E\left(W_{t} \mid\left\{W_{u}, u<t\right\}\right)=\phi\left(W_{t-1}, W_{t-2}, \ldots\right) .
$$

Nonlinear models have been introduced to solve the problem, but they may be hard to identify.

In situations where parametric families cannot be adopted with confidence, nonparametric models offer an alternative. Nonparametric kernel regressors seem attractive because they aim at estimating the regression of the observed process $W_{t}$ on its past values $W_{t-1}, \ldots, W_{t-d}$,

$$
r\left(W_{t-1}, \ldots, W_{t-d}\right)=E\left(W_{t} \mid W_{t-1}, \ldots, W_{t-d}\right)
$$

without requiring strong assumptions on the data generating process. The choice of the number $d$ of lags is however crucial for the following reason. When $d$ is chosen too small, the nonparametric predictions are likely to be far from the optimality, even when the number of observations $n$ increases to infinity. On the other hand when $d$ is large, the method is subject to the so-called curse of dimensionality (the kernel estimator converges at a rate $n^{2 /(4+d)}$ which is low when $d$ is large).

In this work we consider a third approach to the problem of time series prediction, which combines parametric and nonparametric methods. The idea is to utilize ARMA residuals as regressors in the nonparametric approach, to forecast the subsequent behaviour of $W_{t}$. More precisely, we consider two approaches. In the first one we use

$$
\tilde{r}\left(W_{t-1}, \ldots, W_{t-\ell}, \epsilon_{t-1}, \ldots, \epsilon_{t-m}\right)=E\left(W_{t} \mid W_{t-1}, \ldots, W_{t-\ell}, \epsilon_{t-1}, \ldots, \epsilon_{t-m}\right)
$$


as an approximation to the optimal prediction in (1.2), where $\epsilon_{t}=W_{t}-E L\left(W_{t} \mid\left\{W_{u}, u<\right.\right.$ $t\}$ ) denotes the linear innovation of the stationary process $\left(W_{t}\right)$. The use of a nonlinear regression aims to account for the underlying nonlinear structure of $W_{t}$. On the other hand, the use of linear innovations aims to alleviate the effects of the above-mentioned curse of dimensionality. Since the $\epsilon_{t}$ 's are not observable, they are replaced by the residuals of an ARMA model. For this method we will show that the rate of convergence approaches $n^{2 /(4+\ell+m)}$, which seems advantageous compared to the traditional nonparametric regression when $\ell+m<d$. In the second approach we use the decomposition of the optimal prediction in (1.2) as the sum of the optimal linear prediction and the optimal (nonlinear) prediction of the linear innovation process:

$$
E\left(W_{t} \mid\left\{W_{u}, u<t\right\}\right)=E L\left(W_{t} \mid\left\{W_{u}, u<t\right\}\right)+E\left(\epsilon_{t} \mid\left\{\epsilon_{u}, u<t\right\}\right) .
$$

The idea is to estimate the first term parametrically, and the second term nonparametrically, in the right-hand side of (1.5). Again the innovations are replaced by residuals to obtain a feasible predictor. Under slightly different assumptions than in the first method, we will establish the consistency and asymptotic normality of the proposed estimator.

Our main motivation for using residuals in nonparametric estimators is parsimony. It is well-known that, in view of the parsimony principle, the class of ARMA models is preferable to the class of AR models (although both classes are dense in the set of the stationary processes). This is precisely the idea which is behind the approaches based on (1.4) and (1.5). If the same asymptotic precision is achieved with a first regression on a large number of past values and a second regression on only few past values and past linear innovations, it is reasonable to think that, in view of the curse of dimensionality, the second regression will do a better job in finite samples.

The essential difficulty in the derivation of the asymptotic results is that variables depending on a first-step estimator are included in the regressors and, for the method based on (1.5), are also included in the regressand. To cope with this problem, the idea is to interpret the ARMA residuals as noisy innovations, that is innovations that are corrupted by the effect of the parameters estimation. We therefore need to establish general asymptotic results for nonparametric regression based on "noisy time series". Specifically, we consider the case where the time $t$ observation is the sum of the realization of an underlying stochastic process and a disturbance, which is allowed to depend on the sample size $n$. 
The intuition behind this semiparametric method is simple and has obvious connections with numerous methods already proposed in the literature, in particular $i$ ) the combination of forecasts from individual models (see Timmermann (2006) and the references therein), ii) the pre-whitening methods, like the one proposed by Carroll, Linton, Mammen and Xiao (2002) for a regression model with autocorrelated errors, iii) the adaptive estimation methods, like the one proposed by Xu and Phillips (2008) for the inference of AR models with heteroscedasticity of unknown form (see also Phillips and Xu (2005), iv) the convex combination of parametric and nonparametric predictions proposed by Einsporn and Birch (1993) and Burman and Chaudhuri (1994) for possibly misspecified regression models (see also Fan and Ullah (1999)), v) the Model-Robust Regression method proposed by Mays, Birch, and Einsporn (2000), vi) the nonparametric correction factor proposed by Glad (1998). The methods mentioned in $v$ ) and vi) have been developed to protect parametric regressions against a model misspecification, which is not the concern of the present paper, but these two methods are close in spirit to the semiparametric method we consider in the present paper because they combine (additively in Mays et al. (2000) and multiplicatively in Glad (1998)) a parametric fit of the raw data and a nonparametric fit of the parametric residuals. In this sense, the method based on (1.5) can be viewed as a semiparametric method of type iv)-vi) in which the parametric fit is the optimal linear predictor.

Our approach is also related to papers dealing with nonparametric regression, or density estimation, in the presence of measurement error. Recent references on this topic are Carroll, Maca and Ruppert (1999) and Schennach (2004), among others. In contrast to those articles, the measurement error in our framework, that is the difference between the innovation and the residual is not independent from the latter. More importantly, it also depends on the sample size. Finally, it does not only concern the regressors but also the regressand.

The paper is organized as follows. In Section 2 we consider nonparametric density estimation and nonparametric regression for noisy data. Consistency and asymptotic normality are established under mixing assumptions on the unobserved stationary process, and a control of the size of the noise in the data. Section 3 uses these results for Kernel estimators based on ARMA residuals. Hybrid predictors, combining parametric and nonparametric techniques, are studied. In Section 4, their finite sample properties are investigated by means of simulations. An application to stock return data is also presented. 
Concluding remarks are given in Section 5 .

The symbol $\stackrel{\mathcal{L}}{\rightarrow}$ denotes convergence in distribution. For any function $f: \mathbb{R}^{d} \rightarrow \mathbb{R}$, let $D_{i_{1} \ldots i_{k}} f(x)=\left(\partial^{k} f / \partial x_{i_{1}} \ldots \partial x_{i_{k}}\right)(x)$. The notation $o_{P}(1)$ is used for a sequence of random vectors that converge to zero in probability. The notation $R_{n}=O_{P}\left(S_{n}\right)$ means that $R_{n}=S_{n} T_{n}$ for a sequence $T_{n}$ which is bounded in probability.

\section{Kernel estimators applied to noisy data}

In this section we study the behavior of the kernel density and regression estimators when they are computed on noisy data. This will be applied to ARMA residuals, considered as noisy innovations. The section may however have its own interest.

Consider a strictly stationary process $Z=\left(Z_{t}\right)_{t \in \mathbb{Z}}$ where $Z_{t}=\left(Y_{t}^{\prime}, X_{t}^{\prime}\right)^{\prime}$, with $Y_{t} \in \mathbb{R}^{d_{0}}$ and $X_{t} \in \mathbb{R}^{d}$. Let $g: \mathbb{R}^{d_{0}} \rightarrow \mathbb{R}$ be a measurable function. Our goal is to estimate the regression

$$
r(x)=E\left(g\left(Y_{t}\right) \mid X_{t}=x\right)
$$

which is assumed to exist. We do not observe the process $\left(Z_{t}\right)$ but, instead, we have $n$ consecutive noisy observations of the form

$$
\tilde{Z}_{1, n}, \ldots, \tilde{Z}_{n, n} \quad \text { with } \quad \tilde{Z}_{t, n}^{\prime}=\left(\tilde{Y}_{t, n}^{\prime}, \tilde{X}_{t, n}^{\prime}\right):=\left(Y_{t}^{\prime}+V_{t, n}^{\prime}, X_{t}^{\prime}+U_{t, n}^{\prime}\right)
$$

where the $V_{t, n}$ and $U_{t, n}$ are disturbance terms. Observe that the "noise" is present both in the regressand and the regressors.

Let $f=f_{X}, f_{Y}$ and $f_{Z}$ be the densities of $X_{t}, Y_{t}$ and $Z_{t}$. Since the seminal paper by Rosenblatt (1956), kernel estimators have been extensively employed for nonparametrically estimating $f$ and $r$. Given a kernel $K: \mathbb{R}^{d} \rightarrow \mathbb{R}$ and a sequence of scalar bandwidths $\left(b_{n}\right)>0$, the kernel density estimator of $f(x)$ is defined by

$$
\tilde{f}(x)=\frac{1}{n b_{n}^{d}} \sum_{t=1}^{n} K\left(\frac{x-\tilde{X}_{t, n}}{b_{n}}\right) .
$$

When $\tilde{f}(x) \neq 0$, the regression $r(x)$ can be estimated by the Nadaraya-Watson estimator

$$
\tilde{r}(x)=\frac{\tilde{\varphi}(x)}{\tilde{f}(x)}, \quad \tilde{\varphi}(x)=\frac{1}{n b_{n}^{d}} \sum_{t=1}^{n} g\left(\tilde{Y}_{t, n}\right) K\left(\frac{x-\tilde{X}_{t, n}}{b_{n}}\right) .
$$

It will be convenient to consider the pseudo-estimators

$$
\hat{f}(x)=\frac{1}{n b_{n}^{d}} \sum_{t=1}^{n} K\left(\frac{x-X_{t}}{b_{n}}\right), \quad \hat{r}(x)=\frac{\hat{\varphi}(x)}{\hat{f}(x)}, \quad \hat{\varphi}(x)=\frac{1}{n b_{n}^{d}} \sum_{t=1}^{n} g\left(Y_{t}\right) K\left(\frac{x-X_{t}}{b_{n}}\right)
$$


based on the non-observable variables $Z_{1}, \ldots, Z_{n}$.

The main asymptotic properties of the latter estimators, when $Z_{1}, \ldots, Z_{n}$ are observed, are available in the statistical literature (see e.g. the monographs by Prakasa Rao (1983), Fan and Yao (2003)). We start by examining the consistency properties of the density and regression estimators when applied to noisy data.

\subsection{Consistency}

Establishing consistency requires some technical assumptions which we are listing here. Let $\|\cdot\|$ denote any norm on $\mathbb{R}^{d}$ or $\mathbb{R}^{d_{0}}$.

A1: $\quad K$ is a density with respect to the Lebesgue measure, $\int_{\mathbb{R}^{d}} u K(u) d u=0$, $\int_{\mathbb{R}^{d}}\|u\|^{2} K(u) d u<\infty$ and $\lim _{\|u\| \rightarrow \infty}\|u\|^{d} K(u)=0$. In addition $K$ satisfies the Lipschitz condition $|K(u)-K(v)|<C\|u-v\|$ for some constant $C$.

A2: $\quad$ The strong mixing coefficients of the process $Z$, defined by

$$
\alpha_{Z}(k)=\sup _{A \in \sigma\left(Z_{u}, u \leq t\right), B \in \sigma\left(Z_{u}, u \geq t+k\right)}|P(A \cap B)-P(A) P(B)|,
$$

are such that

$$
\sum_{h=0}^{\infty}\left\{\alpha_{Z}(h)\right\}^{\frac{\nu}{2+\nu}}<\infty \text { for some } \nu>0 .
$$

A3: The vector $x \in \mathbb{R}^{d}$ is such that $f(x)>0$. The functions $f_{Z}, f$ and $\varphi:=r f$ are twice derivable with continuous and bounded second order derivatives. We have $E\left|g\left(Y_{t}\right)\right|^{2+\nu}<\infty$, and $\sup _{u} \int|g(y)|^{2+\nu} f_{Z}(y, u) d y<\infty$, where $\nu$ is defined in A2. There exists some constant $C$ such that $\left|g\left(y^{\prime}\right)-g(y)\right|<C\left\|y^{\prime}-y\right\|$ for all $y, y^{\prime} \in \mathbb{R}^{d_{0}}$.

A4: $\quad b_{n} \rightarrow 0 \quad$ and $\quad n b_{n}^{d\left(1+\frac{\nu}{2+\nu}\right)} \rightarrow \infty \quad$ as $n \rightarrow \infty$, for the constant $\nu>0$ involved in $\mathbf{A 2}-\mathbf{A} 3$.

An assumption similar to $\sup _{x} \int|g(y)|^{2+\nu} f_{Z}(y, x) d y<\infty$ is also made in Mack and Silverman (1982). Note that Pham (1986) and Carrasco and Chen (2002) have shown that, for a wide class of processes, the mixing conditions made in Assumption A2 are satisfied.

On the disturbance terms, we make the following assumption.

B1: $\quad$ There exists $\tau<1$ such that

$$
\sum_{t=1}^{n}\left\|U_{t, n}\right\|+\sum_{t=1}^{n}\left\|V_{t, n}\right\|+\sum_{t=1}^{n}\left(\left\|Y_{t}\right\|+\left\|V_{t, n}\right\|\right)\left\|U_{t, n}\right\|=O_{P}\left(n^{\tau}\right) .
$$


We will see that this situation arises with $\tau=1 / 2$ when the kernel estimators are applied to residuals of parametric models.

Theorem 2.1 Under Assumptions A1-A4 and B1, the kernel density and regression estimators based on the noisy observations satisfy

$$
\tilde{f}(x)=f(x)+o_{P}(1) \quad \text { and } \quad \tilde{r}(x)=r(x)+o_{P}(1)
$$

whenever $n b_{n}^{(1+d) /(1-\tau)} \rightarrow \infty$. When $b_{n}=c n^{-1 /\{d+4+d \nu /(2+\nu)\}}, c>0$, and $\tau<$ $\{1+d \nu /(2+\nu)\} /\{d+4+d \nu /(2+\nu)\}$

$$
\begin{aligned}
\tilde{f}(x)-f(x) & =O_{P}\left(n^{-2 /\{d+4+d \nu /(2+\nu)\}}\right), \\
\tilde{r}(x)-r(x) & =O_{P}\left(n^{-2 /\{d+4+d \nu /(2+\nu)\}}\right) .
\end{aligned}
$$

In the proof below we use the fact that under the assumptions of this proposition, except B1, (2.4), (2.5), and (2.6) hold for the pseudo-estimators obtained by replacing $\tilde{f}(x)$ and $\tilde{r}(x)$ by $\hat{f}(x)$ and $\hat{r}(x)$. Such a result is standard and can be obtained under many other assumptions (see e.g. Bosq (1996) or Härdle (1990)). This proposition thus shows that the asymptotic behavior of the kernel estimators is not affected by the presence of small disturbances. Note also that for an exponential mixing rate $\alpha_{Z}(h)=O\left(\rho^{h}\right)$ with $\rho \in(0,1)$, the constant $\nu>0$ can be chosen arbitrarily small, so that the rate of convergence (2.5)(2.6) is arbitrarily close to the optimal rate $O_{P}\left(n^{-2 /(d+4)}\right)$ of the case of identically and independently distributed (iid) variables $\left(Z_{t}\right)$.

Proof. In this proof and the subsequent ones, $C$ denotes a generic positive constant whose exact value is unimportant and may vary from line to line.

Under A1-A4 we have ${ }^{1}$

$$
\hat{f}(x)=f(x)+o_{P}(1) \quad \text { and } \quad \hat{r}(x)=r(x)+o_{P}(1)
$$

and for $b_{n}=c n^{-1 /\{d+4+d \nu /(2+\nu)\}}, c>0$

$$
\max \{\hat{f}(x)-f(x), \hat{r}(x)-r(x)\}=O_{P}\left(n^{-2 /\{d+4+d \nu /(2+\nu)\}}\right) .
$$

\footnotetext{
${ }^{1}$ For the convenience of the reader, and also because we have not been able to find a reference establishing these results with exactly the same assumptions, a complete proof of (2.7)-(2.8) is available from the authors.
} 
Now, by $\mathbf{A} \mathbf{1}$ and $\mathbf{B} \mathbf{1}$ we have

$$
\begin{aligned}
|\tilde{f}(x)-\hat{f}(x)| & \leq \frac{1}{n b_{n}^{d}} \sum_{t=1}^{n}\left|K\left(\frac{x-X_{t}-U_{t, n}}{b_{n}}\right)-K\left(\frac{x-X_{t}}{b_{n}}\right)\right| \\
& \leq \frac{C}{n b_{n}^{d}} \sum_{t=1}^{n}\left\|\frac{U_{t, n}}{b_{n}}\right\|=O_{P}\left(n^{\tau-1} b_{n}^{-d-1}\right) .
\end{aligned}
$$

The right-hand side of the last equality tends to zero when $n b_{n}^{(1+d) /(1-\tau)} \rightarrow \infty$. The first consistency in (2.4) then follows from (2.7) and the triangular inequality

$$
|\tilde{f}(x)-f(x)| \leq|\tilde{f}(x)-\hat{f}(x)|+|\hat{f}(x)-f(x)|
$$

In view of this inequality and (2.8), the optimal rate is reached since $n^{\tau-1} b_{n}^{-d-1}=$ $o\left(n^{-2 /\{d+4+d \nu /(2+\nu)\}}\right)$ for $b_{n}=c n^{-1 /\{d+4+d \nu /(2+\nu)\}}$, which entails (2.5).

We now consider the consistency of the regressor $\tilde{r}(x)=\tilde{\varphi}(x) / \tilde{f}(x)$. First note that (2.7) implies

$$
|\hat{\varphi}(x)-\varphi(x)|=o_{P}(1)
$$

We have

$$
\begin{array}{r}
\tilde{\varphi}(x)-\hat{\varphi}(x)=\frac{1}{n b_{n}^{d}} \sum_{t=1}^{n}\left\{g\left(Y_{t}+V_{t, n}\right)-g\left(Y_{t}\right)\right\} K\left(\frac{x-X_{t}}{b_{n}}\right) \\
+\frac{1}{n b_{n}^{d}} \sum_{t=1}^{n} g\left(Y_{t}+V_{t, n}\right)\left\{K\left(\frac{x-X_{t}-U_{t, n}}{b_{n}}\right)-K\left(\frac{x-X_{t}}{b_{n}}\right)\right\} .
\end{array}
$$

The assumptions made in A1 entail that $\bar{K}:=\sup _{u}|K(u)|<\infty$. By the Lipschitz condition in A3 we have $|g(x)|=|g(x)-g(0)+g(0)| \leq C(\|x\|+1)$. Using A3, we then obtain

$$
\begin{aligned}
|\hat{\varphi}(x)-\tilde{\varphi}(x)| & \leq \frac{C}{n b_{n}^{d}} \sum_{t=1}^{n} K\left(\frac{x-X_{t}}{b_{n}}\right)\left\|V_{t, n}\right\|+\frac{C}{n b_{n}^{d+1}} \sum_{t=1}^{n}\left|g\left(Y_{t}+V_{t, n}\right)\right|\left\|U_{t, n}\right\| \\
& \leq \frac{C \bar{K}}{n b_{n}^{d}} \sum_{t=1}^{n}\left\|V_{t, n}\right\|+\frac{C^{2}}{n b_{n}^{d+1}} \sum_{t=1}^{n}\left(\left\|Y_{t}\right\|+\left\|V_{t, n}\right\|+1\right)\left\|U_{t, n}\right\| .
\end{aligned}
$$

Thus, in view of $\mathbf{B} 1$,

$$
|\hat{\varphi}(x)-\tilde{\varphi}(x)|=O_{P}\left(n^{\tau-1} b_{n}^{-d-1}\right) .
$$

The consistency of the numerator of $\tilde{r}(x)=\tilde{\varphi}(x) / \tilde{f}(x)$ follows from $(2.10)$ and (2.12). The consistency of the denominator has already been established. The second equality of (2.4) then follows by Slutsky's lemma. 
From $(2.8),(2.12)$ and the consistency of $\hat{f}(x)$, we obtain

$$
\begin{aligned}
\tilde{\varphi}(x)-\varphi(x) & =\tilde{\varphi}(x)-\hat{\varphi}(x)+\{\hat{r}(x)-r(x)\} \hat{f}(x)+r(x)\{\hat{f}(x)-f(x)\} \\
& =O_{P}\left(n^{-2 /\{d+4+d \nu /(2+\nu)\}}\right)
\end{aligned}
$$

under the assumptions of the theorem on $b_{n}$ and $\tau$. Under the same conditions,

$$
\begin{aligned}
\tilde{r}(x)-r(x) & =\frac{\tilde{\varphi}(x)-\varphi(x)}{\tilde{f}(x)}+\varphi(x) \frac{f(x)-\tilde{f}(x)}{f(x) \tilde{f}(x)} \\
& =O_{P}\left(n^{-2 /\{d+4+d \nu /(2+\nu)\}}\right)
\end{aligned}
$$

which completes the proof.

\subsection{Asymptotic normality of the regression estimator}

The previous assumptions are strengthened as follows.

A1': Assumption A1 holds and the Kernel function $K$ is three times differentiable with bounded derivatives, and the first two derivatives are integrable.

A2':

$$
\sum_{h=0}^{\infty} h^{\varrho}\left\{\alpha_{Z}(h)\right\}^{\frac{\nu}{2+\nu}}<\infty, \quad \text { where } \nu>0 \text { and } \varrho>\frac{\nu}{2+\nu} .
$$

A3': Assumption A3 holds with $f_{Z}(y, x) \leq C f_{Y}(y)$, for some positive constant $C$, for all $x \in \mathbb{R}^{d}, y \in \mathbb{R}^{d_{0}}$. For all $h>0$, the vector $\left(Z_{t}^{\prime}, Z_{t-h}^{\prime}\right)=\left(Y_{t}^{\prime}, X_{t}^{\prime}, Y_{t-h}^{\prime}, X_{t-h}^{\prime}\right)$ admits a continuous density $f_{Z_{h}, Z_{0}}$ such that $f_{Z_{h}, Z_{0}}(y, x, \tilde{y}, \tilde{x}) \leq C f_{Y_{h}, Y_{0}}(y, \tilde{y})$, for some positive constant $C$, for all $x, \tilde{x} \in \mathbb{R}^{d}, y, \tilde{y} \in \mathbb{R}^{d_{0}}$, where $f_{Y_{h}, Y_{0}}$ denotes the density of $\left(Y_{t}^{\prime}, Y_{t-h}^{\prime}\right)$.

When $f_{Z}(y, x) \leq C f_{Y}(y)$, the function $f_{Z}(y, x)$ is said to be uniformly in the order of $f_{Y}(y)$. Note that this assumption, together with $E\left|g\left(Y_{t}\right)\right|^{2+\nu}<\infty$, entails the condition $\sup _{u} \int|g(y)|^{2+\nu} f_{Z}(y, u) d y<\infty$ in A3. When $Z_{t}$ is gaussian and $Y_{t} \in \mathbb{R}$, one can take

$$
C=(2 \pi)^{-d_{0} / 2}\left|\operatorname{Var}\left(Y_{t}\right)-\operatorname{Cov}\left(Y_{t}, X_{t}\right) \operatorname{Var}\left(X_{t}\right)^{-1} \operatorname{Cov}\left(X_{t}, Y_{t}\right)\right|^{-1 / 2} .
$$

A4': For the constants $\varrho$ and $\nu$ involved in A2' and A3 we have, as $n \rightarrow \infty$,

$$
n b_{n}^{4+d} \rightarrow 0, \quad n b_{n}^{\frac{\nu(4+d)}{\varrho(2+\nu)}} \rightarrow \infty, \quad n b_{n}^{d\left(1+\frac{\nu}{2+\nu}\right)} \rightarrow \infty .
$$


For a real random variable $X$ and a constant $s>0$, let $\|X\|_{s}=\left\{\int|x|^{s} d P(x)\right\}^{1 / s}$. For a random vector $X=\left(X_{1}, \ldots, X_{k}\right)^{\prime}$, let $\|X\|_{s}=\left\|\sum_{i=1}^{k}\left|X_{i}\right|\right\|_{s}$.

B2: There exist nonnegative numbers $\tau_{0}$ and $\tau_{1}$, positive constants $\zeta_{1}, \zeta_{2}, \gamma_{1}, \gamma_{2}, \gamma_{3}$ with $\frac{1}{\zeta_{1}}+\frac{1}{\zeta_{2}}=1$ and $\frac{1}{\gamma_{1}}+\frac{1}{\gamma_{2}}+\frac{1}{\gamma_{3}}=1$, sequences of positive random variables $\left(\mu_{t}\right),\left(\rho_{t}\right)$, $\left(h_{n}\right),\left(k_{n}\right),\left(u_{t, n}\right)$ and $\left(v_{t, n}\right)$, a constant $C>0$ and a constant $\rho \in(0,1)$, a sequence of integers $\left(a_{n}\right)$, such that

$$
a_{n} \rightarrow \infty \quad \text { and } \quad a_{n}=o\left(\sqrt{n b_{n}^{d}}\right) \quad \text { as } \quad n \rightarrow \infty,
$$

and

$$
\left\|V_{t, n}\right\| \leq \rho_{t}+k_{n} v_{t, n}, \quad\left\|U_{t, n}\right\| \leq \mu_{t}+h_{n} u_{t, n}
$$

where

$$
\begin{aligned}
& k_{n}=O_{P}\left(n^{-\tau_{0}}\right), \quad h_{n}=O_{P}\left(n^{-\tau_{1}}\right), \quad \max \left\{\rho_{t}, \mu_{t}\right\} \leq C \rho^{t} \text { a.s. }, \\
& \max \left\{\left\|Y_{t}\right\|_{\zeta_{1}},\left\|v_{t, n}\right\|_{\zeta_{1}},\left\|u_{t, n}^{3}\right\|_{\zeta_{2}},\left\|v_{t, n}\right\|_{\gamma_{1}},\left\|Y_{t}\right\|_{\gamma_{1}},\left\|u_{t, n}^{2}\right\|_{\gamma_{2}}\right\} \leq C .
\end{aligned}
$$

In the following result, we establish the asymptotic distribution of $\hat{r}(x)$ under two sets of assumptions. The first one is simpler, but it cannot be used for the application developed in Section 3 since, as we shall see, the real number involved in $\mathbf{B} 1$ is $\tau=1 / 2$ when the noisy data consist of ARMA residuals.

Theorem 2.2 Assume A1'-A4' and either

1. B1 with $n^{2 \tau-1} b_{n}^{-(2+d)} \rightarrow 0$,

or

2. B2 with, for $\left.k_{\overline{\tau_{0}}+\frac{1}{2}}, b_{n}^{2,}, \frac{1}{\zeta_{2}}-\frac{1}{\zeta_{1}}\right) \rightarrow 0$,

2) $n \rho^{2 a_{n}} b_{n}^{-(2 k+d)+\frac{2 d}{\zeta_{2}}} \rightarrow 0$,

3) $\quad n^{-1} \rho^{2 a_{n}} b_{n}^{-(2 k+d)} \rightarrow 0$,

4) $n^{1-2 k \tau_{1}} b_{n}^{-(2 k+d)+\frac{2 d}{\gamma_{3}}} \rightarrow 0$,

5) $n^{1-2 \tau_{0}} \rho^{2 a_{n}} b_{n}^{-(2 k+d)+\frac{2 d}{\zeta_{2}}} \rightarrow 0$,

6) $n^{1-2\left(\tau_{0}+k \tau_{1}\right)} b_{n}^{-(2 k+d)+\frac{2 d}{\gamma_{3}}} \rightarrow 0$,

7) $\quad n \rho^{2 a_{n}} b_{n}^{-(6+d)} \rightarrow 0$,

8) $n^{1-6 \tau_{1}} b_{n}^{-(6+d)} \rightarrow 0$,

9) $\quad n^{1-2 \tau_{0}} \rho^{2 a_{n}} b_{n}^{-(6+d)} \rightarrow 0$,

10) $n^{1-2\left(\tau_{0}+3 \tau_{1}\right)} b_{n}^{-(6+d)} \rightarrow 0$.

Then, letting $F_{2}(x)=\varphi_{2}(x)-f(x) r^{2}(x)$,

$$
\sqrt{n b_{n}^{d}}\{\tilde{r}(x)-r(x)\} \stackrel{\mathcal{L}}{\rightarrow} \mathcal{N}\left(0, \frac{F_{2}(x)}{f^{2}(x)} \int_{\mathbb{R}^{d}} K^{2}(u) d u\right) .
$$


It should be noted that, under $\mathbf{A} 1^{\prime}-\mathbf{A} 4$ ', the asymptotic distribution of $\hat{r}(x)$ is exactly the same as in (2.15). Hence, the asymptotic behavior of regression estimator is not affected by the presence of a "small" noise in the data.

Before proving this result, we establish the following technical lemma.

Lemma 2.1 Let $\left(X_{t}\right)$ be as in Section 2 and let the density $f$ of $X_{t}$ satisfy A3. Let $\gamma>1$ and $H: \mathbb{R}^{d} \rightarrow \mathbb{R}^{+}$such that $\int_{\mathbb{R}^{d}} H^{\gamma}(t) d t<\infty$, and let $\left(b_{n}\right)$ be a sequence of positive numbers such that $b_{n} \rightarrow 0$ as $n \rightarrow \infty$. Then we have

$$
\left\|H\left(\frac{x-X_{t}}{b_{n}}\right)\right\|_{\gamma}=O\left(b_{n}^{\frac{d}{\gamma}}\right) .
$$

Proof. We have, by the change of variable formula

$$
\left\|H\left(\frac{x-X_{t}}{b_{n}}\right)\right\|_{\gamma}=\left\{\int_{\mathbb{R}^{d}} H^{\gamma}\left(\frac{x-u}{b_{n}}\right) f(u) d u\right\}^{\frac{1}{\gamma}}=\left\{b_{n}^{d} \int_{\mathbb{R}^{d}} H^{\gamma}(t) f\left(x-b_{n} t\right) d t\right\}^{\frac{1}{\gamma}} .
$$

Note that $f$ is bounded under A3. When $n \rightarrow \infty$ the latter integral converges to $f(x) \int_{\mathbb{R}^{d}} H^{\gamma}(t) d t$ by the dominated convergence theorem. The conclusion follows.

Proof of Theorem 2.2. Asymptotic normality of regression estimators under strong mixing assumptions were first established by Robinson (1983). Under A1 and A2'-A4' we have ${ }^{2}$

$$
\sqrt{n b_{n}^{d}}\{\hat{r}(x)-r(x)\} \stackrel{\mathcal{L}}{\rightarrow} \mathcal{N}\left(0, \frac{F_{2}(x)}{f^{2}(x)} \int_{\mathbb{R}^{d}} K^{2}(u) d u\right) .
$$

We have

$$
\hat{r}(x)-\tilde{r}(x)=\frac{\hat{\varphi}(x)-\tilde{\varphi}(x)}{\hat{f}(x)}-\tilde{\varphi}(x) \frac{\hat{f}(x)-\tilde{f}(x)}{\hat{f}(x) \tilde{f}(x)} .
$$

Under B1, by (2.9), (2.12) and (2.17),

$$
\sqrt{n b_{n}^{d}}(\hat{r}(x)-\tilde{r}(x))=O_{P}\left(n^{\tau-1 / 2} b_{n}^{-d / 2-1}\right)=o_{P}(1)
$$

Thus (2.15) follows, in view of (2.16), under the first set of assumptions.

Now suppose that B2 holds. We will show that $\sqrt{n b_{n}^{d}}(\hat{r}(x)-\tilde{r}(x))$ converges to zero in probability, which, by (2.16), will be sufficient to prove (2.15). In view of (2.17) it suffices to prove that

$$
\sqrt{n b_{n}^{d}}|\hat{\varphi}(x)-\tilde{\varphi}(x)|=o_{P}(1) \quad \text { and } \quad \sqrt{n b_{n}^{d}}|\hat{f}(x)-\tilde{f}(x)|=o_{P}(1) .
$$

\footnotetext{
${ }^{2} \mathrm{~A}$ proof is available from the authors.
} 
In view of (2.11) and using $\mathbf{A} \mathbf{3}$ we have

$$
\begin{aligned}
& \sqrt{n b_{n}^{d}}|\hat{\varphi}(x)-\tilde{\varphi}(x)| \leq \frac{C}{\sqrt{n b_{n}^{d}}} \sum_{t=1}^{n} K\left(\frac{x-X_{t}}{b_{n}}\right)\left\|V_{t, n}\right\| \\
& +\frac{C}{\sqrt{n b_{n}^{d}}} \sum_{t=1}^{n}\left|g\left(Y_{t}+V_{t, n}\right)\right|\left|K\left(\frac{x-X_{t}-U_{t, n}}{b_{n}}\right)-K\left(\frac{x-X_{t}}{b_{n}}\right)\right| \\
:= & S_{1}+S_{2} .
\end{aligned}
$$

With the notation $\bar{K}=\sup _{u}|K(u)|$ introduced in the proof of Theorem 2.1, we have

$$
\begin{aligned}
S_{1} & \leq \frac{C}{\sqrt{n b_{n}^{d}}} \sum_{t=1}^{n} K\left(\frac{x-X_{t}}{b_{n}}\right)\left(\rho_{t}+k_{n} v_{t, n}\right) \\
& \leq \frac{C \bar{K}}{\sqrt{n b_{n}^{d}}} \sum_{t=1}^{n} \rho_{t}+\frac{C k_{n}}{\sqrt{n b_{n}^{d}}} \sum_{t=1}^{n} K\left(\frac{x-X_{t}}{b_{n}}\right) v_{t, n} .
\end{aligned}
$$

The first term in the right-hand side of the last inequality converges to 0 in probability by B2 and A4' (implying $n b_{n}^{d} \rightarrow \infty$ ). Moreover, using successively the Hölder inequality, Lemma 2.1 and $\mathbf{B 2}$ we find

$$
\begin{aligned}
& E\left\{\frac{1}{\sqrt{n b_{n}^{d}}} \sum_{t=1}^{n} K\left(\frac{x-X_{t}}{b_{n}}\right) v_{t, n}\right\} \\
\leq & \frac{C}{\sqrt{n b_{n}^{d}}} \sum_{t=1}^{n}\left\|K\left(\frac{x-X_{t}}{b_{n}}\right)\right\|_{\zeta_{2}}\left\|v_{t, n}\right\|_{\zeta_{1}} \\
\leq & \frac{C b_{n}^{d / \zeta_{2}}}{\sqrt{n b_{n}^{d}}} \sum_{t=1}^{n}\left\|v_{t, n}\right\|_{\zeta_{1}}=O\left(n^{1 / 2} b_{n}^{\frac{d}{2}\left(\frac{1}{\zeta_{2}}-\frac{1}{\zeta_{1}}\right)}\right) .
\end{aligned}
$$

From B2 and 1) in (2.14) we deduce that the second term in (2.21) converges to 0 in probability. It follows that

$$
S_{1}=o_{P}(1)
$$

To handle $S_{2}$ we will make a third-order Taylor expansion of the Kernel function around $\left(x-X_{t}\right) / b_{n}$. Write $U_{t, n}=\left(U_{1, t, n}, \ldots, U_{d, t, n}\right)^{\prime}$. We have

$$
\begin{aligned}
K\left(\frac{x-X_{t}-U_{t, n}}{b_{n}}\right)= & K\left(\frac{x-X_{t}}{b_{n}}\right)-\frac{1}{b_{n}} \sum_{i=1}^{d} U_{i, t, n} D_{i} K\left(\frac{x-X_{t}}{b_{n}}\right) \\
& +\frac{1}{2 b_{n}^{2}} \sum_{i, j=1}^{d} U_{i, t, n} U_{j, t, n} D_{i j} K\left(\frac{x-X_{t}}{b_{n}}\right) \\
& -\frac{1}{6 b_{n}^{3}} \sum_{i, j, k=1}^{d} U_{i, t, n} U_{j, t, n} U_{k, t, n} D_{i j k} K\left(\frac{x-X_{t, n}}{b_{n}}\right),
\end{aligned}
$$


where $X_{t, n}$ is between $X_{t}$ and $X_{t}+U_{t, n}$. Thus, using the vector norm $\|a\|=\sum\left|a_{i}\right|$,

$$
\begin{aligned}
& \left|K\left(\frac{x-X_{t}-U_{t, n}}{b_{n}}\right)-K\left(\frac{x-X_{t}}{b_{n}}\right)\right| \leq \frac{1}{b_{n}}\left\|U_{t, n}\right\| \sum_{i=1}^{d}\left|D_{i} K\left(\frac{x-X_{t}}{b_{n}}\right)\right| \\
& +\frac{1}{2 b_{n}^{2}}\left\|U_{t, n}\right\|^{2} \sum_{i, j=1}^{d}\left|D_{i j} K\left(\frac{x-X_{t}}{b_{n}}\right)\right|+\frac{1}{6 b_{n}^{3}}\left\|U_{t, n}\right\|^{3} \sum_{i, j, k=1}^{d}\left|D_{i j k} K\left(\frac{x-X_{t, n}}{b_{n}}\right)\right| .
\end{aligned}
$$

Hence, with the elementary inequality $(|a|+|b|)^{k} \leq 2^{k}\left(|a|^{k}+|b|^{k}\right)$,

$$
\begin{aligned}
S_{2} \leq & \frac{C}{\sqrt{n b_{n}^{d}}} \sum_{t=a_{n}}^{n} \frac{1}{b_{n}}\left|g\left(Y_{t}+V_{t, n}\right)\right|\left(\mu_{t}+h_{n} u_{t, n}\right) \sum_{i=1}^{d}\left|D_{i} K\left(\frac{x-X_{t}}{b_{n}}\right)\right| \\
& +\frac{C}{\sqrt{n b_{n}^{d}}} \sum_{t=a_{n}}^{n} \frac{1}{b_{n}^{2}}\left|g\left(Y_{t}+V_{t, n}\right)\right|\left(\mu_{t}^{2}+h_{n}^{2} u_{t, n}^{2}\right) \sum_{i, j=1}^{d}\left|D_{i j} K\left(\frac{x-X_{t}}{b_{n}}\right)\right| \\
& +\frac{C}{\sqrt{n b_{n}^{d}}} \sum_{t=a_{n}}^{n} \frac{1}{b_{n}^{3}}\left|g\left(Y_{t}+V_{t, n}\right)\right|\left(\mu_{t}^{3}+h_{n}^{3} u_{t, n}^{3}\right) \sum_{i, j, k=1}^{d}\left|D_{i j k} K\left(\frac{x-X_{t, n}}{b_{n}}\right)\right| \\
& +\frac{C}{\sqrt{n b_{n}^{d}}} \sum_{t=1}^{a_{n}}\left(1+\left\|Y_{t}\right\|+\left\|V_{t, n}\right\|\right) \\
:= & S_{21}+S_{22}+S_{23}+S_{24},
\end{aligned}
$$

where the last term follows from the fact that the kernel function is bounded and from the Lipschitz condition on $g$. We have

$$
\begin{aligned}
S_{21} \leq & \frac{C}{b_{n} \sqrt{n b_{n}^{d}}} \sum_{t=a_{n}}^{n}\left(1+\left\|Y_{t}\right\|+\rho_{t}\right) \mu_{t} \sum_{i=1}^{d}\left|D_{i} K\left(\frac{x-X_{t}}{b_{n}}\right)\right| \\
& +\frac{C h_{n}}{b_{n} \sqrt{n b_{n}^{d}}} \sum_{t=a_{n}}^{n}\left(1+\left\|Y_{t}\right\|+\rho_{t}\right) u_{t, n} \sum_{i=1}^{d}\left|D_{i} K\left(\frac{x-X_{t}}{b_{n}}\right)\right| \\
& +\frac{C k_{n}}{b_{n} \sqrt{n b_{n}^{d}}} \sum_{t=a_{n}}^{n} v_{t, n} \mu_{t} \sum_{i=1}^{d}\left|D_{i} K\left(\frac{x-X_{t}}{b_{n}}\right)\right| \\
& +\frac{C h_{n} k_{n}}{b_{n} \sqrt{n b_{n}^{d}}} \sum_{t=a_{n}}^{n} v_{t, n} u_{t, n} \sum_{i=1}^{d}\left|D_{i} K\left(\frac{x-X_{t}}{b_{n}}\right)\right| \\
:= & S_{211}+h_{n} S_{212}+k_{n} S_{213}+h_{n} k_{n} S_{214} .
\end{aligned}
$$

Because the derivatives of $K$ are bounded and $\rho_{t}$ and $\mu_{t}$ are $O\left(\rho^{t}\right)$ with probability one, we have

$$
S_{211} \leq \frac{C \rho^{a_{n}}}{b_{n} \sqrt{n b_{n}^{d}}} \sum_{t=a_{n}}^{n}\left\|Y_{t}\right\| \sum_{i=1}^{d}\left|D_{i} K\left(\frac{x-X_{t}}{b_{n}}\right)\right|+O_{P}\left(\frac{\rho^{a_{n}}}{b_{n} \sqrt{n b_{n}^{d}}}\right) .
$$

Denote by $S_{211}^{*}$ the first term of the right-hand side of this equality. It is easy to check that A1' entails $\int\left|D_{i} K(t)\right|^{\gamma} d t<\infty$ for any power $\gamma \geq 1$. Using Lemma 2.1 and by the 
Hölder inequality, we have

$$
\begin{aligned}
E\left(S_{211}^{*}\right) & \leq \frac{C \rho^{a_{n}}}{b_{n} \sqrt{n b_{n}^{d}}} \sum_{t=a_{n}}^{n}\left\|Y_{t}\right\|_{\zeta_{1}} \sum_{i=1}^{d}\left\|D_{i} K\left(\frac{x-X_{t}}{b_{n}}\right)\right\|_{\zeta_{2}} \\
& \leq \frac{C \rho^{a_{n}} b_{n}^{\frac{d}{\zeta_{2}}}}{b_{n} \sqrt{n b_{n}^{d}}} \sum_{t=a_{n}}^{n}\left\|Y_{t}\right\|_{\zeta_{1}} \leq \frac{C \rho^{a_{n}} n b_{n}^{\frac{d}{\zeta_{2}}}}{b_{n} \sqrt{n b_{n}^{d}}}=o(1),
\end{aligned}
$$

where the last equality follows from 2$)$ in (2.14) with $k=1$. Thus, noting that $S_{211}^{*} \geq 0$, we have $S_{211}^{*}=o_{P}(1)$. Using 3) with $k=1$ and (2.25) we then obtain $S_{211}=o_{P}(1)$. By Lemma 2.1 and the Hölder inequality, we have

$$
\begin{aligned}
E\left(S_{212}\right) & \leq \frac{1}{b_{n} \sqrt{n b_{n}^{d}}} \sum_{t=a_{n}}^{n}\left(1+\left\|Y_{t}\right\|_{\gamma_{1}}+\left\|\rho_{t}\right\|_{\gamma_{1}}\right)\left\|u_{t, n}\right\|_{\gamma_{2}} \sum_{i=1}^{d}\left\|D_{i} K\left(\frac{x-X_{t}}{b_{n}}\right)\right\|_{\gamma_{3}} \\
& \leq \frac{C b_{n}^{-1-\frac{d}{2}+\frac{d}{\gamma_{3}}}}{\sqrt{n}} \sum_{t=a_{n}}^{n}\left(1+\left\|Y_{t}\right\|_{\gamma_{1}}+C \rho^{t}\right)\left\|u_{t, n}\right\|_{\gamma_{2}}=O\left(b_{n}^{-1-\frac{d}{2}+\frac{d}{\gamma_{3}}} \sqrt{n}\right) .
\end{aligned}
$$

Thus, 4) with $k=1$ in (2.14) entails $h_{n} S_{212}=o_{P}(1)$. For the same reasons, 5) with $k=1$ entails $k_{n} S_{213}=o_{P}(1)$, and 6) with $k=1$ entails $h_{n} k_{n} S_{214}=o_{P}(1)$. Thus, in view of (2.24), we have shown that $S_{21}=o_{P}(1)$. By exactly the same arguments, 2)-6) with $k=2$

entail $S_{22}=o_{P}(1)$. For $S_{23}$ we use the boundedness of the third derivatives (A1') and conclude similarly using the convergence 7 )-10) in (2.14). Finally,

$$
S_{24} \leq \frac{C}{\sqrt{n b_{n}^{d}}} \sum_{t=1}^{a_{n}}\left(1+\left\|Y_{t}\right\|+\rho_{t}\right)+\frac{C k_{n}}{\sqrt{n b_{n}^{d}}} \sum_{t=1}^{a_{n}}\left(1+\left\|Y_{t}\right\|+v_{t, n}\right):=S_{241}+k_{n} S_{242} .
$$

We have

$$
\left\|S_{24 i}\right\|_{1} \leq \frac{C a_{n}}{\sqrt{n b_{n}^{d}}}=o(1), \quad i=1,2
$$

which proves that $S_{24 i}=o_{P}(1)$ and thus that $S_{24}=o_{P}(1)$. Therefore we have

$$
S_{2}=o_{P}(1)
$$

In view of (2.22) and (2.20) this proves that the first equality in (2.19) holds. The second equality follows along the same lines and the proof of Theorem 2.2 is complete.

\section{Kernel estimators applied to ARMA residuals}

Many non linear processes admit ARMA representations (see e.g. Romano and Thombs (1996), Francq, Roy and Zakoïan (2005) and the references therein). This is not very 
surprising because, from the Wold theorem, any purely non deterministic second-order stationary process $\left(X_{t}\right)$ has an infinite MA representation, which can be closely approximated by finite order ARMA models. The noise in these ARMA representations is only the linear innovation of $\left(X_{t}\right)$ and is not an iid sequence (otherwise $\left(X_{t}\right)$ would be a linear process). These representations are referred to as weak ARMA representations, by opposition to the standard strong ARMA representations where the noise is supposed to be iid.

In this section we will show how the weak ARMA residuals can be used in nonparametric predictors. Density estimators based on residuals of time series models have already been studied by Robinson (1983) and Liebscher (2001), among others. Such residual-based estimators can be used to obtain adaptive estimators (see Drost Klaassen and Werker, 1997) and to obtain $\sqrt{n}$-consistent plug-in estimators for functionals of a density (see Schick and Wefelmeyer, 2004). Our framework here is quite different, since we study the asymptotic behavior of kernel estimators of autoregressions when lagged values of ARMA residuals are taken as explanatory variables (in Section 3.2) and when the ARMA residuals constitute the dependent variable (in Section 3.3).

\subsection{Weak ARMA residuals}

We now introduce the assumptions we need. Let $W=\left(W_{t}\right)_{t \in \mathbb{Z}}$ be a real second-order stationary $\operatorname{ARMA}(p, q)$ process such that

$$
W_{t}+\sum_{i=1}^{p} \phi_{i} W_{t-i}=\epsilon_{t}+\sum_{i=1}^{q} \psi_{i} \epsilon_{t-i}, \quad \forall t \in \mathbb{Z} .
$$

The parameter $\theta_{0}=\left(\phi_{1}, \ldots, \phi_{p}, \psi_{1}, \ldots, \psi_{q}\right)^{\prime}$ is unknown and we observe a sample $W_{1}, W_{2}, \ldots, W_{n}$ of $W$. For any $\theta=\left(\theta_{1}, \ldots, \theta_{p+q}\right)^{\prime} \in \mathbb{R}^{p+q}$, define two polynomials by $\Phi_{\theta}(z)=1+\theta_{1} z+\cdots+\theta_{p} z^{p}$ and $\Psi_{\theta}(z)=1+\theta_{p+1} z+\cdots+\theta_{p+q} z^{q}$. For any $\delta>0$, let the compact set

$$
\Theta_{\delta}=\left\{\theta \in \mathbb{R}^{p+q} ; \text { the zeros of } \Phi_{\theta}(z) \text { and } \Psi_{\theta}(z) \text { have moduli } \geq 1+\delta\right\}
$$

We make the following assumptions.

C1. $\epsilon=\left(\epsilon_{t}\right)$ is a strictly stationary sequence of uncorrelated random variables with zero mean and variance $\sigma^{2}>0$. 
C2. $\theta_{0}$ belongs to the interior of $\Theta_{\delta}$, and the polynomials $\Phi_{\theta_{0}}(z)$ and $\Psi_{\theta_{0}}(z)$ have no zero in common.

C3. $\phi_{p}$ and $\psi_{q}$ are not both equal to zero (by convention $\phi_{0}=\psi_{0}=1$ ).

The sequence of the $\alpha$-mixing (strong mixing) coefficients of some process $(U)=\left(U_{t}\right)_{t \in \mathbb{Z}}$ is denoted by $\left\{\alpha_{U}(h)\right\}_{h \geq 0}$. We will consider, alternatively, the following mixing assumptions.

C4. The process $(W, \epsilon)$ satisfies Model (3.1), the moment condition $E\left|W_{t}\right|^{4+2 \nu}<\infty$ (or equivalently $\left.E\left|\epsilon_{t}\right|^{4+2 \nu}<\infty\right)$ and the mixing condition

$$
\sum_{h=0}^{\infty}\left\{\alpha_{W, \epsilon}(h)^{\frac{\nu}{2+\nu}}<\infty \quad \text { for some } \nu>0 .\right.
$$

C5. The process $(W, \epsilon)$ satisfies Model (3.1), with $E\left|W_{t}\right|^{4+2 \nu}<\infty$ and

$$
\sum_{h=0}^{\infty}\left\{\alpha_{\epsilon}(h)\right\}^{\frac{\nu}{2+\nu}}<\infty \quad \text { for some } \nu>0 .
$$

For all $\theta \in \Theta$, let $\epsilon_{t}(\theta)=\Psi_{\theta}^{-1}(B) \Phi_{\theta}(B) W_{t}$ and $e_{t}(\theta)=\Psi_{\theta}^{-1}(B) \Phi_{\theta}(B)\left(W_{t} 1_{1 \leq t \leq n}\right)$, where $B$ denotes the backshift operator. Note that $\epsilon_{t}(\theta)$ is not computable from the sample, but it is introduced for theoretical purpose. Let $\hat{\theta}_{n}$ be a Least Squares Estimator (LSE) satisfying, almost surely,

$$
Q_{n}\left(\hat{\theta}_{n}\right)=\min _{\theta \in \Theta_{\delta}} Q_{n}(\theta) \quad \text { where } \quad Q_{n}(\theta)=\frac{1}{2 n} \sum_{t=1}^{n} e_{t}^{2}(\theta) .
$$

The $\operatorname{ARMA}(p, q)$ residuals are then defined by $\hat{\epsilon}_{t}=e_{t}\left(\hat{\theta}_{n}\right)$ for $t=1, \ldots, n$. Francq and Zakoïan (1998) have shown that the LSE is strongly consistent and asymptotically normal under C1-C4. We will need the additional technical lemma, giving the behavior of the weak ARMA residuals.

Lemma 3.1 If C1-C3 and either $\mathbf{C 4}$ or $\mathbf{C 5}$ hold then

$$
\hat{\epsilon}_{t}=\epsilon_{t}+s_{t}+O_{P}\left(n^{-1 / 2}\right) \quad \text { with } \quad\left|s_{t}\right| \leq C \rho^{t}
$$

where the constants $\rho \in(0,1)$ and $C$ only depend on the initial values $W_{0}, \ldots, W_{1-p}, \epsilon_{0}$, $\ldots, \epsilon_{1-q}$. Moreover $\sum_{t=1}^{n}\left|\epsilon_{t}-\hat{\epsilon}_{t}\right|=O_{P}\left(n^{1 / 2}\right)$.

Proof. Write $s_{t}=s_{t}\left(\theta_{0}\right)$, where $s_{t}(\theta)=e_{t}(\theta)-\epsilon_{t}(\theta)$. A Taylor expansion of $e_{t}(\cdot)$ around $\theta_{0}$ yields

$$
\hat{\epsilon}_{t}=e_{t}\left(\hat{\theta}_{n}\right)=s_{t}+\epsilon_{t}\left(\theta_{0}\right)+\left(\hat{\theta}_{n}-\theta_{0}\right)^{\prime} \frac{\partial e_{t}}{\partial \theta}\left(\theta^{*}\right),
$$


where $\theta^{*}$ is between $\hat{\theta}_{n}$ and $\theta_{0}$. It is shown in Francq and Zakoïan (1998, Lemma 1 and Theorem 2, and 2000, Lemmas A.1 and A.2) that $\sup _{\theta \in \Theta}\left|s_{t}(\theta)\right| \leq C \rho^{t}$, where $C$ is a measurable function of the initial values, that $\frac{\partial e_{t}}{\partial \theta_{j}}(\theta)=\sum_{i=1}^{t-1} c_{i, j}(\theta) W_{t-i}$ with $c_{i}:=$ $\max _{j \in\{1, \ldots, p+q\}} \sup _{\theta \in \Theta}\left\|c_{i, j}(\theta)\right\|=O\left(\rho^{i}\right)$, and that $\sqrt{n}\left(\hat{\theta}_{n}-\theta_{0}\right)=O_{P}(1)$ under C4. The same results can be obtained following the same lines of proof under C5. The proof of (3.3) follows. Now

$$
\sum_{t=1}^{n}\left|\epsilon_{t}-\hat{\epsilon}_{t}\right| \leq \sum_{t=1}^{n}\left|s_{t}\right|+\left\|\hat{\theta}_{n}-\theta_{0}\right\| \sum_{t=1}^{n} \sum_{i \geq 1} c_{i}\left|W_{t-i}\right|,
$$

and the conclusion follows from the $\sqrt{n}$-consistency of $\hat{\theta}_{n}$ and the fact that $E \sum_{t=1}^{n} \sum_{i \geq 1} c_{i}\left|W_{t-i}\right| \leq C n \sum_{i \geq 1} \rho^{i}=O(n)$.

\subsection{Nonparametric prediction based on past observables and ARMA residuals}

Because we will now consider different regressions, we need to modify the notation of Section 2 for the regression functions $r(x)$ and $\tilde{r}(x)$. Let $\ell$ and $m$ be two integers such that $d:=\ell+m \neq 0$. Recall that the $\hat{\epsilon}_{t}$ are the residuals of the LSE of the weak $\operatorname{ARMA}(p, q)$ model (3.1). With some abuse of notation and obvious conventions, write $\tilde{r}\left\{W_{t} \mid\left(W_{t-1}, \ldots, W_{t-\ell}, \hat{\epsilon}_{t-1}, \ldots, \hat{\epsilon}_{t-m}\right)=x\right\}$ for the kernel estimator of the regression of $W_{t}$ on $W_{t-1}, \ldots, W_{t-\ell}, \hat{\epsilon}_{t-1}, \ldots, \hat{\epsilon}_{t-m}$ evaluated at $x=\left(x_{1}, \ldots, x_{d}\right)^{\prime}$. For any real sequence $\left(X_{t}\right)$ and $k<\ell$, let $\mathbf{X}_{t-k}^{t-\ell}=\left(X_{t-k}, X_{t-k-1}, \ldots, X_{t-\ell}\right)$.

Our first main result in this section is the following, showing that under mild regularity conditions, a kernel regression on ARMA residuals is equivalent to a (theoretical) regression on (non observed) linear innovations.

Theorem 3.1 Assume that A1 and $\mathbf{C 1 - C 4}$ hold true and that for all integers $d_{1}$ and $d_{2}$, and all indices $t_{1}, \ldots, t_{d_{1}}, t_{1}^{\prime}, \ldots, t_{d_{1}}^{\prime}$ of $\mathbb{Z}$, the vector $\left(W_{t_{1}}, \ldots, W_{t_{d_{1}}}, \epsilon_{t_{1}^{\prime}}, \ldots, \epsilon_{t_{d_{2}}^{\prime}}\right)$ has a strictly positive density $f_{Z}$ which is uniformly in the order of each of its marginal densities. Assume also that the functions $f_{Z}\left(z_{1}, \ldots, z_{d_{1}+d_{2}}\right)$ and $\left(z_{2}, \ldots, z_{d_{1}+d_{2}}\right) \mapsto$ $\int z_{1} f_{Z}\left(z_{1}, \ldots, z_{d_{1}+d_{2}}\right) d z_{1}$ are twice derivable, with continuous and bounded second order derivatives. Then

(i) If $b_{n} \rightarrow 0$ and $n b_{n}^{2(1+d)} \rightarrow \infty$ as $n \rightarrow \infty$,

$$
\tilde{r}\left\{W_{t} \mid\left(\mathbf{W}_{t-1}^{t-\ell}, \hat{\boldsymbol{\epsilon}}_{t-1}^{t-m}\right)=x\right\} \rightarrow E\left\{W_{t} \mid\left(\mathbf{W}_{t-1}^{t-\ell}, \boldsymbol{\epsilon}_{t-1}^{t-m}\right)=x\right\}, \quad \text { in probability. }
$$


(ii) If, in addition, A1', A2' and $\mathbf{A} 4$ ' hold with $Z=(W, \epsilon)$, if $E\left|W_{t}\right|^{s}<\infty$ with $s \geq 4+\nu$ and $s>6 d /(d-2), d>2$, and if $n^{2} b_{n}^{6+d} \rightarrow \infty$ hold,

$$
\begin{aligned}
& \sqrt{n b_{n}^{d}}\left[\tilde{r}\left\{W_{t} \mid\left(\mathbf{W}_{t-1}^{t-\ell}, \hat{\boldsymbol{\epsilon}}_{t-1}^{t-m}\right)=x\right\}-E\left\{W_{t} \mid\left(\mathbf{W}_{t-1}^{t-\ell}, \boldsymbol{\epsilon}_{t-1}^{t-m}\right)=x\right\}\right] \\
\stackrel{\mathcal{L}}{\rightarrow} & \mathcal{N}\left(0, \frac{\operatorname{Var}\left\{W_{t} \mid\left(\mathbf{W}_{t-1}^{t-\ell}, \boldsymbol{\epsilon}_{t-1}^{t-m}\right)=x\right\}}{f(x)} \int_{\mathbb{R}^{d}} K^{2}(u) d u\right)
\end{aligned}
$$

where $f$ is the density of $\left(\mathbf{W}_{t-1}^{t-\ell}, \boldsymbol{\epsilon}_{t-1}^{t-m}\right)$.

Concerning the assumptions, the following remark can be made. For a purely non deterministic strong ARMA process with innovations admitting a positive density over $\mathbb{R}$, the vector $\left(W_{t}, \ldots, W_{t-\ell}, \epsilon_{t-1}, \ldots, \epsilon_{t-m}\right)$ has also a positive density. This a not necessarily the case for a general weak ARMA model (3.1) because the distribution of $W$ is not entirely defined by the distribution of $\epsilon$ and the ARMA coefficients.

Proof. To use the results of Section 2 we set

$$
Z_{t}=\left(\mathbf{W}_{t}^{t-\ell}, \boldsymbol{\epsilon}_{t-1}^{t-m}\right)^{\prime}, \quad \tilde{Z}_{t, n}=\left(\mathbf{W}_{t}^{t-\ell}, \hat{\boldsymbol{\epsilon}}_{t-1}^{t-m}\right)^{\prime}, \quad U_{t, n}=\left(0, \ldots, 0, \boldsymbol{\epsilon}_{t-1}^{t-m}-\hat{\boldsymbol{\epsilon}}_{t-1}^{t-m}\right)^{\prime},
$$

$V_{t, n}=0$, and $Y_{t}=W_{t}$. Using (3.4), we obtain

$$
\begin{aligned}
& \sum_{t=1}^{n}\left\{\left\|U_{t, n}\right\|+\left\|V_{t, n}\right\|+\left(\left\|Y_{t}\right\|+\left\|V_{t, n}\right\|\right)\left\|U_{t, n}\right\|\right\} \\
\leq & \sum_{j=1}^{m} \sum_{t=1}^{n}\left\{C \rho^{t}+\left\|\hat{\theta}_{n}-\theta_{0}\right\| \sum_{i \geq 1} c_{i}\left|W_{t-j-i}\right|\right\}\left(1+\left|W_{t}\right|\right) \\
= & O_{P}\left(n^{1 / 2}\right) .
\end{aligned}
$$

Thus B1 holds with $\tau=1 / 2$. Because $\alpha_{Z}(h) \leq \alpha_{W, \epsilon}(h-\max \{\ell, m\})$, Assumption $\mathbf{A} 2$ is satisfied. Assumption A3 is satisfied because of conditions assumed in the proposition. Since $d(1+\nu /(2+\nu))<2(1+d)$ for all $\nu>0, \mathbf{A} 4$ is satisfied. Then, the convergence of probability follows from (2.4) of Theorem 2.1.

Now we turn to the asymptotic normality. In view of Theorem 2.2, it suffices to verify that assumptions A3', B2 and (2.14) are satisfied. Assumption A3' is directly implied by the conditions made in the proposition. Let us turn to B2. Obviously one can take $\rho_{t}=k_{n}=v_{t, n}=0$ in (2.13). Thus $\tau_{0}$ can be chosen arbitrarily large. To derive the second inequality of (2.13), let us write, as in the proof of Lemma 3.1,

$$
\hat{\epsilon}_{t}-\epsilon_{t}=s_{t}+\left(\hat{\theta}_{n}-\theta_{0}\right)^{\prime} \frac{\partial e_{t}}{\partial \theta}\left(\theta^{*}\right)=s_{t}-\frac{\partial Q_{n}}{\partial \theta^{\prime}}\left(\theta_{0}\right) J_{n}^{-1} \frac{\partial e_{t}}{\partial \theta}\left(\theta^{*}\right)
$$


where the matrix $J_{n}=\left[\frac{\partial^{2}}{\partial \theta \partial \theta^{\prime}} Q_{n}\left(\theta_{n, i j}^{*}\right)\right]$ is non-singular for sufficiently large $n$, the $\theta_{n, i j}^{*}$ and $\theta^{*}$ being between $\theta_{0}$ and $\hat{\theta}_{n}$. Thus, using the multiplicativity of the norm $\|A\|=\sum\left|a_{i j}\right|$, we obtain

$$
\left|\hat{\epsilon}_{t}-\epsilon_{t}\right| \leq\left|s_{t}\right|+\left\|\frac{\partial Q_{n}}{\partial \theta^{\prime}}\left(\theta_{0}\right)\right\|\left\|J_{n}^{-1}\right\|\left\|\frac{\partial e_{t}}{\partial \theta}\left(\theta^{*}\right)\right\|
$$

and thus

$$
\left\|U_{t, n}\right\| \leq \sum_{j=1}^{m}\left|s_{t-j}\right|+\left\|J_{n}^{-1}\right\|\left\|\frac{\partial Q_{n}}{\partial \theta^{\prime}}\left(\theta_{0}\right)\right\| \sum_{j=1}^{m}\left\|\frac{\partial e_{t-j}}{\partial \theta}\left(\theta^{*}\right)\right\|=\mu_{t}+h_{n} u_{t, n}
$$

with $\mu_{t}=\sum_{j=1}^{m}\left|s_{t-j}\right|, h_{n}=\left\|J_{n}^{-1}\right\|\left\|\frac{\partial Q_{n}}{\partial \theta}\left(\theta_{0}\right)\right\|$ and $u_{t, n}=\sum_{j=1}^{m}\left\|\frac{\partial e_{t-j}}{\partial \theta}\left(\theta^{*}\right)\right\|$. It is shown in Francq and Zakoïan (1998) that $\sqrt{n} \frac{\partial Q_{n}}{\partial \theta}\left(\theta_{0}\right)$ converges in distribution to a non degenerated gaussian distribution and that $J_{n}$ converges almost surely to a non-singular matrix $J$. Therefore $h_{n}=O_{P}\left(n^{-1 / 2}\right)$, and one can take $\tau_{1}=1 / 2$. Using arguments given in the proof of Lemma 3.1 and the Minkowski inequality we obtain

$$
\left\|\frac{\partial e_{t}}{\partial \theta}\left(\theta^{*}\right)\right\|_{\zeta} \leq \sum_{i=1}^{\infty} \sum_{j=1}^{p+q} \sup _{\theta \in \Theta}\left|c_{i, j}(\theta)\right|\left\|W_{t-i}\right\|_{\zeta} \leq C,
$$

for all $\zeta$ such that $E\left|W_{t}\right|^{\zeta}<\infty$. We deduce that the moment conditions of $\mathbf{B 2}$ are satisfied if $W_{t}$ admits moments of order $\zeta_{1}, 3 \zeta_{2}, \gamma_{1}$ and $2 \gamma_{2}$. Since $\zeta_{1}=3 \zeta_{2}$ with $\zeta_{1}^{-1}+\zeta_{2}^{-1}=1$ if and only if $\zeta_{1}=4$, a moment of order 4 , at least, is required for $W_{t}$.

Because $\tau_{0}$ can be chosen arbitrarily large, the conditions 1),6), 9) and 10) in (2.14) are always satisfied.

With $a_{n}=\left[(\log n)^{2}\right]$ (the integer part of $\left.(\log n)^{2}\right)$ we have $n^{k} \rho^{a_{n}} \rightarrow 0$ as $n \rightarrow \infty$ for all $k$. Thus 2), 3), 5) and 7) in (2.14) are also always satisfied. Because $n b_{n}^{2} \rightarrow \infty$ it is easy to see that Condition 4) with $k=1$ entails Condition 4) with $k=2$. Condition 4) with $k=1$ is satisfied when $\gamma_{3}<2 d /(2+d)$. Since $\gamma_{3}$ must also be strictly greater than 1 , this is only possible when $d>2$. Taking $\gamma_{1}=2 \gamma_{2}$ the required moment condition is $E\left|W_{t}\right|^{3 \gamma_{3} /\left(\gamma_{3}-1\right)}<\infty$. Note that the minimum of the function $\gamma_{3} \mapsto 3 \gamma_{3} /\left(\gamma_{3}-1\right)$ is $6 d /(d-2)$ on $(1,2 d /(2+d))$. It is thus possible to find a suitable $\gamma_{3}$ when $W_{t}$ admits moments of order greater than $6 d /(d-2)$. Condition 8) is satisfied when $n^{2} b_{n}^{6+d} \rightarrow \infty$, which completes the proof. 


\subsection{Linear prediction plus nonlinear prediction of ARMA residuals}

An alternative estimator can be constructed as follows. Note that under C2, $\left(\epsilon_{t}\right)$ is the linear innovation process of $\left(W_{t}\right)$, that is

$$
W_{t}=E L\left(W_{t} \mid\left\{W_{u}, u<t\right\}\right)+\epsilon_{t}
$$

and that the $\sigma$-fields generated by $\left\{W_{u}, u<t\right\}$ and $\left\{\epsilon_{u}, u<t\right\}$ coincide. It follows that (1.5) holds true. Let $\hat{W}_{t}^{L}$ denote the linear prediction of $W_{t}$ based on the estimated ARMA model,

$$
\hat{W}_{t}^{L}=-\sum_{i=1}^{t-1} \hat{\pi}_{i} W_{t-i}, \quad \text { where } \quad \sum_{i=0}^{\infty} \hat{\pi}_{i} z^{i}=\Psi_{\hat{\theta}_{n}}^{-1}(z) \Phi_{\hat{\theta}_{n}}(z), \quad|z| \leq 1 .
$$

Write $\tilde{r}(x)=\tilde{r}\left\{\hat{\epsilon}_{t} \mid\left(\hat{\epsilon}_{t-1}, \ldots, \hat{\epsilon}_{t-d}\right)=x\right\}$ for the kernel estimator of the regression of $\hat{\epsilon}_{t}$ on $\hat{\epsilon}_{t-1}, \ldots, \hat{\epsilon}_{t-d}$ evaluated at $x=\left(x_{1}, \ldots, x_{d}\right)$. The use of

$$
\tilde{W}_{t}=\hat{W}_{t}^{L}+\tilde{r}\left(\hat{\epsilon}_{t-1}, \ldots, \hat{\epsilon}_{t-d}\right)
$$

as an estimator of $E\left(W_{t} \mid\left\{W_{u}, u<t\right\}\right)$ is legitimated by the following result.

Theorem 3.2 Let A1, C1-C3 and C5 hold true. For all integer $d_{1}$, the vector $\left(\epsilon_{t}, \ldots, \epsilon_{t-d_{1}}\right)$ is assumed to have a strictly positive density $f_{\epsilon}$ which is uniformly in the order of each of its marginal densities, and the functions $f_{\epsilon}\left(x_{1}, \ldots, x_{d_{1}+1}\right)$ and $\left(x_{2}, \ldots, x_{d_{1}+1}\right) \mapsto \int x_{1} f_{\epsilon}\left(x_{1}, \ldots, x_{d_{1}+1}\right) d x_{1}$ are supposed to be twice derivable, with continuous and bounded second order derivatives. Then

(i) If $b_{n} \rightarrow 0$ and $n b_{n}^{2(1+d)} \rightarrow \infty$,

$$
\tilde{r}\left\{\hat{\epsilon}_{t} \mid \hat{\boldsymbol{\epsilon}}_{t-1}^{t-d}=x\right\} \rightarrow E\left\{\epsilon_{t} \mid \boldsymbol{\epsilon}_{t-1}^{t-d}=x\right\}, \quad \text { in probability, as } n \rightarrow \infty .
$$

(ii) If, in addition, A1', A2' and $\mathbf{A} 4$ ' hold with $Z=\epsilon$, if $d>2$ and $E\left|W_{t}\right|^{s}<\infty$ with $s \geq 4+\nu$ and $s>6 d /(d-2)$, if $n^{2} b_{n}^{6+d} \rightarrow \infty$ hold,

$$
\sqrt{n b_{n}^{d}}\left[\tilde{r}\left\{\hat{\epsilon}_{t} \mid \hat{\boldsymbol{\epsilon}}_{t-1}^{t-d}=x\right\}-E\left\{\epsilon_{t} \mid \boldsymbol{\epsilon}_{t-1}^{t-d}=x\right\}\right] \stackrel{\mathcal{L}}{\rightarrow} \mathcal{N}\left(0, \frac{\operatorname{Var}\left\{\epsilon_{t} \mid \boldsymbol{\epsilon}_{t-1}^{t-d}=x\right\}}{f(x)} \int_{\mathbb{R}^{d}} K^{2}(u) d u\right)
$$

where $f$ is the density of $\boldsymbol{\epsilon}_{t-1}^{t-d}$.

Note that the assumptions are slightly weaker than those of Theorem 3.1, since no assumption is made on the density or the mixing coefficients of $(W, \epsilon)$. Note also that 
when $\left(\epsilon_{t}\right)$ is a strong noise, $f_{\epsilon}$ is uniformly in the order of its marginal densities if and only if $\epsilon_{t}$ admits a bounded density.

Proof. We now set $Z_{t}=\boldsymbol{\epsilon}_{t}^{t-d}, Y_{t}=\epsilon_{t}, \tilde{Z}_{t, n}=\hat{\boldsymbol{\epsilon}}_{t}^{t-d}, V_{t, n}=\epsilon_{t}-\hat{\epsilon}_{t}, U_{t, n}=\boldsymbol{\epsilon}_{t-1}^{t-d}-\hat{\boldsymbol{\epsilon}}_{t-1}^{t-d}$. Thus

$$
\begin{aligned}
& \sum_{t=1}^{n}\left\{\left\|U_{t, n}\right\|+\left\|V_{t, n}\right\|+\left(\left\|Y_{t}\right\|+\left\|V_{t, n}\right\|\right)\left\|U_{t, n}\right\|\right\} \\
\leq & C \sum_{t=1}^{n} \rho^{t}+\left\|\hat{\theta}_{n}-\theta_{0}\right\| \sum_{t=1}^{n} \sum_{i \geq 1} c_{i}\left|W_{t-i}\right|+\sum_{j=1}^{d} \sum_{t=1}^{n}\left\{C \rho^{t}+\left\|\hat{\theta}_{n}-\theta_{0}\right\| \sum_{i \geq 1} c_{i}\left|W_{t-j-i}\right|\right\} \\
& \times\left\{1+\left|\epsilon_{t}\right|+C \rho^{t}+\left\|\hat{\theta}_{n}-\theta_{0}\right\| \sum_{i \geq 1} c_{i}\left|W_{t-i}\right|\right\} \\
= & O_{P}\left(n^{1 / 2}\right),
\end{aligned}
$$

by arguments already used to prove (3.4). In particular we used the fact that $E\left|\epsilon_{t}\right|\left|W_{t^{\prime}}\right|$ are $E\left|W_{t}\right|\left|W_{t^{\prime}}\right|$ finite for any $t, t^{\prime}$. We also argue that the LSE is $\sqrt{n}$-consistent under C5 (see Francq and Zakoïan, 1998). The consistency follows as in the proof of Theorem 3.1.

To show the asymptotic normality, an adaptation of the proof of Theorem 3.1 is needed. One can take $\left\|V_{t, n}\right\| \leq \rho_{t}+k_{n} v_{t, n}$ with $\rho_{t}=\left|s_{t}\right|, k_{n}=\left\|J_{n}^{-1}\right\|\left\|\frac{\partial Q_{n}}{\partial \theta}\left(\theta_{0}\right)\right\|$ and $v_{t, n}=$ $\left\|\frac{\partial e_{t}}{\partial \theta}\left(\theta^{*}\right)\right\|$, and we still have $\left\|U_{t, n}\right\| \leq \mu_{t}+h_{n} u_{t, n}$ with $\mu_{t}=\sum_{j=1}^{d}\left|s_{t-j}\right|, h_{n}=k_{n}$ and $u_{t, n}=\left\|\sum_{j=1}^{d} \frac{\partial e_{t-j}}{\partial \theta}\left(\theta^{*}\right)\right\|$. The arguments given in the proof of Theorem 3.1 then show that one can take $\tau_{0}=\tau_{1}=1 / 2$ in $\mathbf{B 2}$, and that the moment conditions of $\mathbf{B 2}$ are satisfied if $W_{t}$ admits moments of orders $\zeta_{1}, 3 \zeta_{2}, \gamma_{1}$ and $2 \gamma_{2}$.

Convergence 1) in (2.14) only requires the condition $\zeta_{1}>2$, which can be satisfied when $W_{t}$ admits a moment of order greater than 4. By already given arguments, conditions 2), $3), 5), 7)$ and 9) in (2.14) are always satisfied. Moreover, Condition 4) is satisfied when $\gamma_{3}<2 d /(2+d)$, which requires $d>2$ and the moment condition $E\left|W_{t}\right|^{s}<\infty$ with $s>6 d /(d-2)$. Noting that Condition 6$)$ is entailed by Condition 4, and Condition 10) by Condition 8), we conclude as in the proof of Theorem 3.1.

\subsection{Implementation}

For simplicity, assume that $W_{1-d}, \ldots, W_{n}$ is observed, and consider the one-step ahead prediction of $W_{n+1}$. Three predictors of $W_{n+1}$ can be investigated:

1) the purely nonparametric estimator

$$
\hat{W}_{n+1}^{N P}=\hat{r}\left(W_{n}, \ldots, W_{n-d+1}\right),
$$


where $\hat{r}$ is defined by (2.3), replacing $X_{t}$ by $\left(W_{t-1}, \ldots, W_{t-d}\right), Y_{t}$ by $W_{t}$ and $g$ by the identity function;

2) the purely parametric estimator $\hat{W}_{n+1}^{L}$ defined by (3.5);

3) the mixed predictor of Section 3.3

$$
\hat{W}_{n+1}^{M}=\hat{W}_{n+1}^{L}+\tilde{r}\left(\hat{\epsilon}_{n}, \ldots, \hat{\epsilon}_{n-d+1}\right)
$$

where the $\hat{\epsilon}_{t}$ 's are the ARMA residuals and $\tilde{r}$ is defined by $(2.2)$, replacing $\tilde{X}_{t, n}$ by $\left(\hat{\epsilon}_{t-1}, \ldots, \hat{\epsilon}_{t-d}\right), \tilde{Y}_{t, n}$ by $\hat{\epsilon}_{t}$ and $g$ by the identity function.

The mixed predictor of Section 3.2 could be implemented as well, but for the numerical illustrations we have chosen to concentrate on that of Section 3.3.

\subsection{Testing the nullity of the autoregression function of the linear inno- vation process}

Note that if the observed process $\left(W_{t}\right)$ is a strong ARMA model, or more generally if the linear innovation process $\left(\epsilon_{t}\right)$ of $\left(W_{t}\right)$ is a sequence of martingale differences, then the following null hypothesis holds :

$$
H_{0}: \quad r\left(\epsilon_{t-1}, \ldots, \epsilon_{t-d}\right):=E\left(\epsilon_{t} \mid \epsilon_{t-1}, \ldots, \epsilon_{t-d}\right) \equiv 0 .
$$

It is important to test for $H_{0}$ because, if $H_{0}$ holds then the mixed predictor defined in 3 ) of Section 3.4 has no chance to improve the purely linear predictor 2). Conversely, when $H_{0}$ is rejected the linear model is not optimal in terms of prediction mean squared error (MSE), and it is worth considering the alternative predictors 1) and 3).

The problem of testing a particular specification of a regression against a nonparametric alternative has been intensively studied in the literature (for recent references see Gao and Tong (2002), Hall and Yatchewa (2005), and the references therein). For iid observations, Härdle and Mammen (1993) proposed a goodness-of-fit test based on a distance between a Nadaraya-Watson estimator and the specification of the regression under the null. Kreiss, Neumann and Yao (2008) (denoted by KNY hereafter) extended the test to a time series context. If the linear innovations were observed, the test statistic of KNY would be in our framework

$$
S_{n}=\int_{\mathbb{R}^{d}}\left\{\frac{1}{n b_{n}^{d}} \sum_{t=1}^{n} \epsilon_{t} K\left(\frac{x-\left(\epsilon_{t-1}, \ldots, \epsilon_{t-d}\right)}{b_{n}}\right)\right\}^{2} \omega(x) d x
$$


where $\omega(\cdot)$ is a weight function, which is $\omega(\cdot) \equiv 1$ in the forthcoming applications. Because the $\epsilon_{t}$ are not observed, it is natural to replace $S_{n}$ by the statistic

$$
\tilde{S}_{n}=\int_{\mathbb{R}^{d}}\left\{\frac{1}{n b_{n}^{d}} \sum_{t=1}^{n} \hat{\epsilon}_{t} K\left(\frac{x-\left(\hat{\epsilon}_{t-1}, \ldots, \hat{\epsilon}_{t-d}\right)}{b_{n}}\right)\right\}^{2} \omega(x) d x
$$

where the $\hat{\epsilon}_{t}$ 's are the ARMA residuals obtained in the purely parametric prediction step. It is clear that the kernel and bandwidth involved in (3.6) are not necessarily the same as those involved in the mixed and purely nonparametric predictors (but in our applications we employed the same parameters). Note that when $\omega(\cdot) \equiv 1^{3}$ and the kernel $K$ is the gaussian density, we simply have

$$
\tilde{S}_{n}=\frac{1}{2^{d} \pi^{d / 2} n^{2} b_{n}^{d}} \sum_{t=1}^{n} \sum_{s=1}^{n} \hat{\epsilon}_{t} \hat{\epsilon}_{s} \prod_{i=1}^{d} \exp \left(-\frac{\left(\hat{\epsilon}_{t-i}-\hat{\epsilon}_{s-i}\right)^{2}}{4 b_{n}^{2}}\right) .
$$

KNY showed that, under a set of regularity conditions, the asymptotic distribution of $n b_{n}^{d / 2}\left(S_{n}-E S_{n}\right)$ is gaussian under the null. The approximation of the finite-sample distribution of $S_{n}$ by a normal distribution is however too crude in practice, and Härdle and Mammen (1993) and KNY implement the wild bootstrap to determine the critical values of their tests. We employed exactly the same resampling scheme as in KNY to obtain the critical value $t_{\alpha}^{*}$ of a test of critical region $\left\{\tilde{S}_{n}>t_{\alpha}^{*}\right\}$. More precisely, conditionally on $\hat{\epsilon}_{1}, \ldots, \hat{\epsilon}_{n}$, the critical value $t_{\alpha}^{*}$ is defined as the $(1-\alpha)$ quantile of the distribution of the bootstrap statistic

$$
S_{n}^{*}=\int_{\mathbb{R}^{d}}\left\{\frac{1}{n b_{n}} \sum_{t=1}^{n} \xi_{t} \hat{\epsilon}_{t} K\left(\frac{x-\left(\hat{\epsilon}_{t-1}, \ldots, \hat{\epsilon}_{t-d}\right)}{b_{n}}\right)\right\}^{2} \omega(x) d x,
$$

where the $\xi_{t}$ 's are iid $\mathcal{N}(0,1)$, and are independent of the $\hat{\epsilon}_{t}$ 's.

\section{$4 \quad$ Numerical illustrations}

We first investigate the performance of the three procedures presented in Section 3.4 on simulated data. Then we present an illustration to the prediction of the volatility of stock market returns.

\subsection{Monte Carlo experiments}

We propose an illustrative example based on a chaotic process (see May (1976)). Let

$$
\epsilon_{t}=u_{t}-\frac{1}{2}+\eta_{t}, \quad u_{t}=4 u_{t-1}\left(1-u_{t-1}\right), \quad t \geq 1
$$

\footnotetext{
${ }^{3}$ With constant weights, the test enjoys the property of scale invariance.
} 
where $u_{0}$ has the arc-sinus density $f(x)=\pi^{-1}\{x(1-x)\}^{-1 / 2}$ on the interval $[0,1],\left(\eta_{t}\right)_{t \geq 1}$ is an iid sequence, independent of $u_{0}$, with mean 0 and finite variance $\sigma_{\eta}^{2}$. Since $f$ is the invariant density of $\left(u_{t}\right)$, this process is stationary. We have $E \epsilon_{t}=0$ and, since $u_{t}$ and $1-u_{t}$ have the same law,

$$
\begin{aligned}
\operatorname{Cov}\left(\epsilon_{t}, \epsilon_{t-1}\right) & =\operatorname{Cov}\left(u_{t}, u_{t-1}\right) \\
& =\operatorname{Cov}\left\{4 u_{t-1}\left(1-u_{t-1}\right), u_{t-1}\right\}=\operatorname{Cov}\left(u_{t}, 1-u_{t-1}\right)=0 .
\end{aligned}
$$

The same symmetry argument shows that $\operatorname{Cov}\left(\epsilon_{t}, \epsilon_{t-h}\right)=0$ for all $h \neq 0$. Therefore $\left(\epsilon_{t}\right)$ is a white noise. Consequently, given $\left\{\epsilon_{u}, u \leq t\right\}$, the best linear predictor of $\epsilon_{t+h}$ is equal to zero, for any horizon $h$. However, in general, the best (nonlinear) predictor is quite different. For illustrative purpose, Figure 1 displays the scatter plot of the pairs $\left(\epsilon_{t-1}, \epsilon_{t}\right)$, for $t=1, \ldots, 1000$, obtained by simulation, and the nonlinear regression obtained by tedious computation, in the case where $\eta_{t}$ is uniformly distributed over $[-0.6,-0.4]$. This example illustrates the, possibly dramatic, differences between linear and nonlinear predictions of a given weak ARMA process. One can interpret the ratio

$$
\tau:=\frac{\operatorname{Var} u_{t}}{\operatorname{Var} \epsilon_{t}}=\frac{\operatorname{Var} u_{t}}{\operatorname{Var} u_{t}+\sigma_{\eta}^{2}}=\frac{\frac{1}{8}}{\frac{1}{8}+\sigma_{\eta}^{2}}
$$

as the proportion of the deterministic part in the noise.

To illustrate our method, we will therefore simulate for different values of $\theta$ the MA(1) process $W_{t}=\epsilon_{t}-\theta \epsilon_{t-1}$, where the noise $\left(\epsilon_{t}\right)$ is given by (4.1). We will compare three predictors: the purely nonparametric predictor defined in 1 ) of Section 3.4 with $d=1$, the MA(1) predictor, and the mixed predictor defined in 3) Section 3.4. For the implementation of the nonparametric predictors we used the function sm.autoregression() contained in the package sm of the statistical software R (see http://cran.r-project.org/). We simulated $N=50$ independent replications of a simulation of length $n+m$ of the MA(1) process $W_{t}$. For each replication, the first $n=500$ simulated values served to adjust the 3 predictors, and the last $m=100$ values were used to compare the actual simulated values and their onestep ahead predictions. Figure 2 compares the distributions of the $N m=5000$ prediction errors obtained with the 3 predictors. As expected, the parametric estimator is slightly superior to the purely nonparametric one when $|\theta|$ is large and the deterministic proportion $\tau$ is not too important (lower panel), whereas the purely nonparametric predictor is often more accurate than the MA(1) predictor when $\tau$ is high (upper panels). The distribution of 
the purely nonparametric predictor presents however more extreme values. In all situations considered in Figures 2, the mixed predictor is always more accurate and seems to cumulate the advantages of the linear and nonparametric predictors. To summarize, the predictor can be ranked, in decreasing order of efficiency, as

$$
\hat{W}^{M} \succ \hat{W}^{L} \succ \hat{W}^{N P} \quad \text { when }|\theta| \sim 1 \text { and } \tau \ll 1
$$

and $\hat{W}^{M} \succ \hat{W}^{N P} \succ \hat{W}^{L}$ otherwise. The simulations clearly show the superiority of our approach on this model.

\subsection{Volatility prediction for stock market returns}

Our application concerns daily returns of the following world stock market indices : BEL 20 (Brussels), CAC 40, DAX, FTSE, HSI (Hong Kong), Nikkei, NSE (India), SMI (Swiss), IGBM (Madrid), SP500, SP TSX (Toronto) and SSE (Shanghai), from January 2, 1991 to July 3, 2009 (except for the indices for which such historical data do not exist). The number of observations varies from $n=1130$ for the BEL 20 index (which begins on February 11, 2005) to $n=4591$ for the HSI index. Standard models for such financial series are weak white noises of the form $r_{t}=\sigma_{t} \eta_{t}$ where $r_{t}$ is the log-return, $\eta_{t}$ is an iid noise, with variance equal to 1 , and $\sigma_{t}^{2}$ is the so-called volatility. For the GARCH-type models, $\sigma_{t}$ is a measurable function of $\left\{r_{s}, s<t\right\}$.

In the sequel, we compare the prediction of the volatility (that is the prediction of the squared returns) obtained with the parametric, nonparametric, and mixed methods of Section 3.4. ${ }^{4}$

Denote by $W_{t}, t=1, \ldots, n$ the sequence of the squared returns. In a first set of experiments, for the purely linear predictor, as well as for the parametric component of the mixed predictor, a MA(1) model has been chosen. Results not reported here, based on the test of Section 3.5, show that the assumption that the best predictor is provided by the MA(1) model is clearly rejected on the data.

To compare the effective predictions of the different methods, a part of the observations is used to fit the predictors and a part is reserved for forecasting exercises. Consider the case when $n$ is an even number of the form $n=2 k$ (the case when $n$ is odd is handled

\footnotetext{
${ }^{4}$ For the purely nonparametric predictor, and also for the nonparametric component of the mixed predictor, we used the default implementation of the $\mathrm{R}$ function sm.autoregression().
} 
similarly). For $t=1, \ldots, k$, we used $W_{t}, \ldots, W_{t+k-1}$ to define predictors of $W_{t+k}$ with the methods 1)-3) of Section 3.4. Table 1 indicates that the root mean squared error (RMSE) of prediction is lower with the mixed method for all asset.

In a second set of experiments, we use an $\operatorname{ARMA}(1,1)$ as parametric predictor of $W_{t}$, both for the purely parametric and mixed methods. Note that an $\operatorname{ARMA}(1,1)$ for the squared returns is obtained when the returns follow a $\operatorname{GARCH}(1,1)$, that is the most widely-used model for such financial series.

The first four columns of Table 2 give the $p$-values of the KNY test that the regression of $W_{t}$ on $W_{t-1}, \ldots, W_{t-d}$ is constant. This assumption is clearly rejected. The next four columns give the $p$-values of the KNY-type test of the null hypothesis that the best predictions of the $\operatorname{ARMA}(1,1)$ residuals by $d$ past values are identically equal to zero. One can see that the assumption that the $\operatorname{ARMA}(1,1)$ model is optimal is rejected for five series at least for one $d$. Table 3 gives the prediction RMSE of the different methods. For the series for which the tests of Table 2 do not reject the assumption that the best predictor is $\operatorname{ARMA}(1,1)$, the purely linear prediction is indeed the best, in general. The mixed predictor can however improve the purely linear predictor when the tests of Section 3.5 reject the assumption that the $\operatorname{ARMA}(1,1)$ predictor is optimal. Finally, note that the purely non parametric method is always far from the optimal method.

\section{Conclusion}

The basic idea behind the semiparametric method studied in this paper is to improve linear parametric predictions by predicting non parametrically what is not linearly predictable. We considered two approaches using ARMA models to capture the linear part of the process, and nonparametric regressions involving ARMA residuals to capture the nonlinear part. In order to avoid the curse of dimensionality inherent to nonparametric estimation, a small number of regressors seems reasonable, whereas the orders $p$ and $q$ of the parametric model are allowed to be relatively large. Compared to a purely nonparametric regressor of the form $E\left(X_{t} \mid X_{t-1}, \ldots, X_{t-d}\right)$, such a mixed method presents the advantage of being able to take into account mid-term linear dynamics. This mixed method could thus be worth considering for time series whose dynamics can not be well taken into account by a very small number of lagged values of the observed process (as it is the case for MA and 
mixed ARMA models or for seasonal models) and, at the same time, exhibit nonlinearities. This method could be also of interest to distinguish or compare the purely linear and the purely nonlinear dynamics.

In this paper regularity conditions were given for consistency and asymptotic normality of the residual-based nonparametric regressor. We established intermediate results which are also applicable in a more general context of triangular arrays of noisy observations. We presented simulation experiments, and an illustration to the volatility prediction of 12 stock market indices, in which the mixed method outperforms both the linear predictor and the purely nonparametric predictor.

The $\mathrm{R}$ code used for the numerical illustrations is available on the web pages of the authors.

\section{References}

Bosq, D. (1996) Nonparametric Statistics for Stochastic Processes. Estimation and Prediction. Lecture Notes in Statist 110, Springer Verlag.

Burman, P. and P. Chaudhuri (1994) A hybrid approach to parametric and nonparametric regression Technical Report No. 243, Division of Statistics, University of California Davis.

Carrasco, M. and X. Chen (2002) Mixing and moment properties of various GARCH and stochastic volatility models. Econometric Theory 18, 17-39.

Carroll, R.J., Linton, O., Mammen, E. and Z. Xiao, (2002) More Efficient Kernel Estimation in Nonparametric Regression with Autocorrelated Errors, STICERD - Econometrics Paper Series /2002/435, Suntory and Toyota International Centres for Economics and Related Disciplines, LSE.

Carroll, R.J., Maca, J.D., and D. Ruppert (1999) Nonparametric regression in the presence of measurement error. Biometrika 86, 541-554.

Drost, F.C., Klaassen, C.A.J., and B.J.M. Werker (1997) Adaptive estimation in timeseries models. Annals of Statistics 25, 786-817.

Einsporn, R.L., and J.B. Birch (1993) Model robust regression: using nonparametric regression to improve parametric regression analyses. Technical Report 93-5, Department of Statistics, Virginia Polytechnic Institute and State University, Blacksburg, $V A$.

Fan, Y. and A. Ullah (1999) Asymptotic Normality of a Combined Regression Estimator. Journal of Multivariate Analysis 71, 191-240

Fan, J. and Q. Yao (2003) Nonlinear Time Series: Nonparametric and Parametric Methods. Springer. 
Francq, C., Roy, R. and J-M. Zakoïan (2005) Goodness-of-fit Tests for ARMA Models with Uncorrelated Errors. Journal of the American Statististical Association 100, $532-544$.

Francq, C. and J-M. Zakoïan (1998) Estimating Linear Representations of Nonlinear Processes. Journal of Statistical Planning and Inference 68, 145-165.

Francq, C. and J-M. Zakoïan (2000) Covariance matrix estimation for estimators of mixing Wold's ARMA. Journal of Statistical Planning and Inference 83, 369-394.

Glad, I. (1998) Parametrically guided non-parametric regression Scandinavian Journal of Statistics 25, 649-668.

Hall, P. and A. Yatchewa (2005) Unified approach to testing functional hypotheses in semiparametric contexts. Journal of Econometrics 127, 225-252.

Gao, J. and H. Tong (2002) Model Specification Tests in Nonparametric Stochastic Regression Models. Journal of Multivariate Analysis 83, 324-359 (2002)

Härdle, W. (1990) Applied Nonparametric Regression. Cambridge University Press: Cambridge.

Härdle, W. and E. Mammen (1993) Comparing nonparametric versus parametric regression fits. Annals of Statistics 21, 1926-1947.

Kreiss, J.P., Neumann, M. H and Q.W. Yao (2008) Bootstrap tests for simple structures in nonparametric time series regression. Statistics and its interface 1 367-380.

Liebscher, E. (2001) Estimation of the density and the regression function under mixing conditions. Statistics \& Decisions 19, 9-26.

Mack, Y.P. and B.W. Silverman (1982) Weak and strong uniform consistency of kernel regression estimates. Z. Wahrscheinlichkeitsth. verw. Geb. 61, 405-415.

May, R.M. (1976) Simple mathematical models with very complicated dynamics. Nature 261, 459-467.

Mays, J., Birch, J.B. and R. Einsporn (2000) An overview of model-robust regression. Journal of Statistical Computation and Simulation 66, 79-100.

Pham, D.T. (1986) The mixing property of bilinear and generalised random coefficients autoregressive models. Stochastic Processes and their Applications 23, 291-300.

Phillips, P.C.B. and K.-L. Xu (2005) Inference in Autoregression under Heteroskedasticity. Journal of Time Series Analysis 27, 289-308.

Prakasa Rao, B.L.S. (1983) Nonparametric Functional Estimation. Academic Press, NewYork.

Robinson, P.M. (1983) Nonparametric estimators for time series. Journal of Time Series Analysis 4, 185-207.

Romano, J.P. and L.A. Thombs (1996) Inference for Autocorrelations under Weak Assumptions. Journal of the American Statististical Association 91, 590-600. 
Rosenblatt, M. (1956) Remarks on Some Nonparametric Estimates of a Density Function. Annals of Mathematical Statistics 27 832-835.

Schennach, S.M. (2004) Nonparametric regression in the presence of measurement error. Econometric Theory 20, 1046-1093.

Schick, A. and W. Wefelmeyer (2004) Root n consistent and optimal density estimators for moving average processes. Scandinavian Journal of Statistics 31, 63-78.

Timmermann, A. (2006) Forecast Combination. Handbook of Economic Forecasting, Vol.1, ed. Elliott G., Granger C. and Timmermann A., Amsterdam: North-Holland, 135-194.

Xu, K.-L. and P.C.B. Phillips (2008) Adaptive Estimation of Autoregressive Models with Time-Varying Variances. Journal of Econometrics 142, 265-280. 


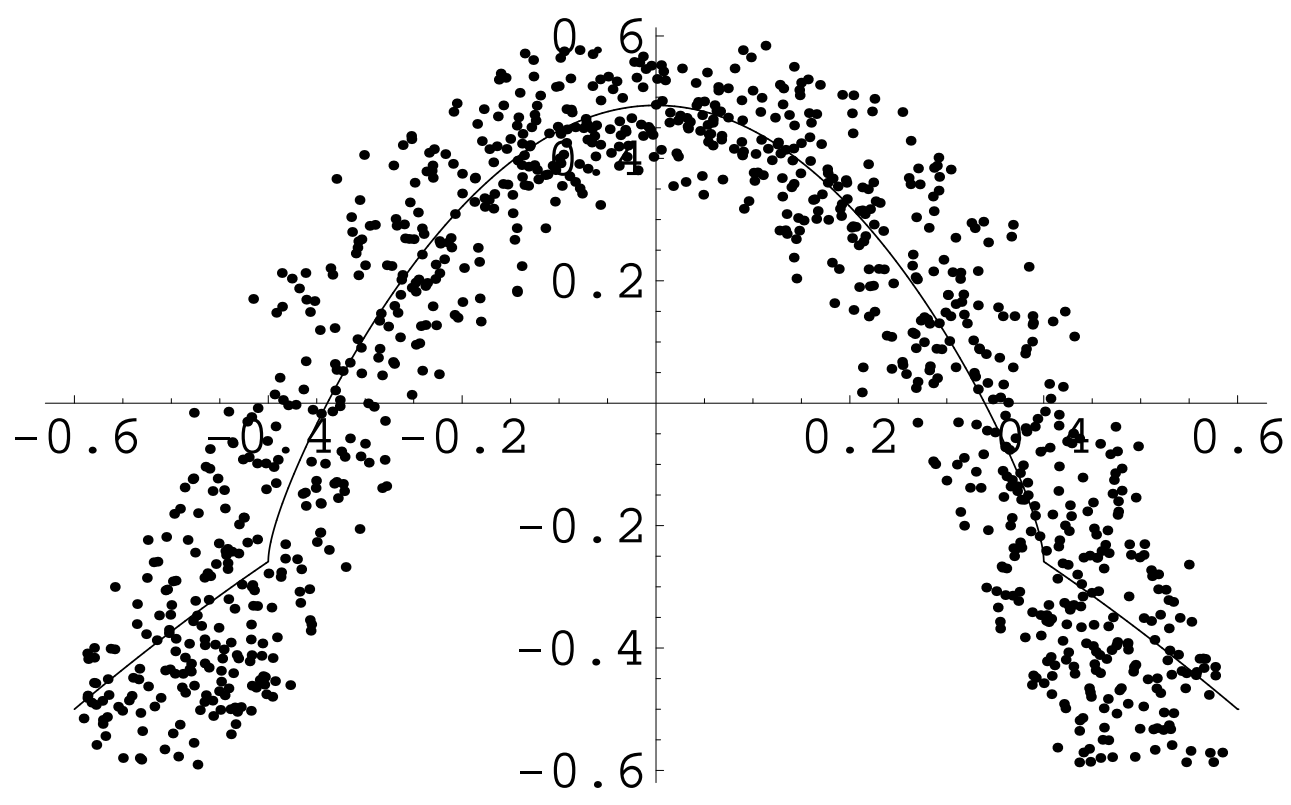

Figure 1: Scatter plot of 1,000 pairs $\left(\epsilon_{t}, \epsilon_{t-1}\right)$, simulated from (4.1) with $\eta_{t}$ uniformly distributed over $[-0.6,-0.4]$. The full line is the theoretical (nonlinear) regression of $\epsilon_{t}$ on $\epsilon_{t-1}$.

Table 1: RMSE of the MA(1), nonparametric (NP) and mixed (Mixed) predictions. For each series, the smallest RMSE is underlined.

\begin{tabular}{lrrrrrrrrr}
\hline \hline & MA(1) & \multicolumn{9}{c}{ NP } \\
& & $d=1$ & $d=2$ & $d=3$ & $d=4$ & $d=1$ & $d=2$ & $d=3$ & $d=4$ \\
BEL & 8.365 & 9.165 & 8.901 & 8.592 & 8.856 & 8.446 & $\underline{8.274}$ & 8.387 & 8.435 \\
CAC & 6.739 & 6.764 & 6.767 & 6.746 & 6.635 & 6.720 & 6.631 & 6.617 & $\underline{6.604}$ \\
DAX & 7.131 & 7.133 & 7.077 & 6.973 & 6.949 & 7.044 & 6.948 & 6.886 & $\underline{6.871}$ \\
FTSE & 5.229 & 5.246 & 5.234 & 5.230 & 5.236 & 5.174 & $\underline{5.074}$ & 5.146 & 5.149 \\
HSI & 9.100 & 9.359 & 9.244 & 9.177 & 9.189 & 8.879 & 8.861 & $\underline{8.775}$ & 8.786 \\
Nikkei & 7.971 & 7.973 & 7.975 & 7.961 & 7.975 & 7.904 & $\underline{7.888}$ & 7.902 & 7.910 \\
NSE & 13.790 & 13.693 & 13.545 & 13.654 & 13.540 & 13.705 & $\underline{13.532}$ & 13.587 & 13.557 \\
SMI & 4.845 & 4.974 & 5.025 & 5.017 & 4.948 & 4.794 & 4.776 & 4.764 & $\underline{4.742}$ \\
IGBM & 7.694 & 7.777 & 8.392 & 8.242 & 7.792 & $\underline{7.556}$ & 7.636 & 7.631 & 7.663 \\
SP500 & 6.232 & 6.244 & 6.292 & 6.349 & 6.261 & $\underline{6.141}$ & 6.142 & 6.158 & 6.195 \\
SPTSX & 6.634 & 6.963 & 7.061 & 7.214 & 7.232 & 6.620 & 6.666 & 6.630 & $\underline{6.607}$ \\
SSE & 8.476 & 8.625 & 8.628 & 8.563 & 8.596 & 8.565 & 8.534 & 8.481 & $\underline{8.435}$ \\
\hline
\end{tabular}



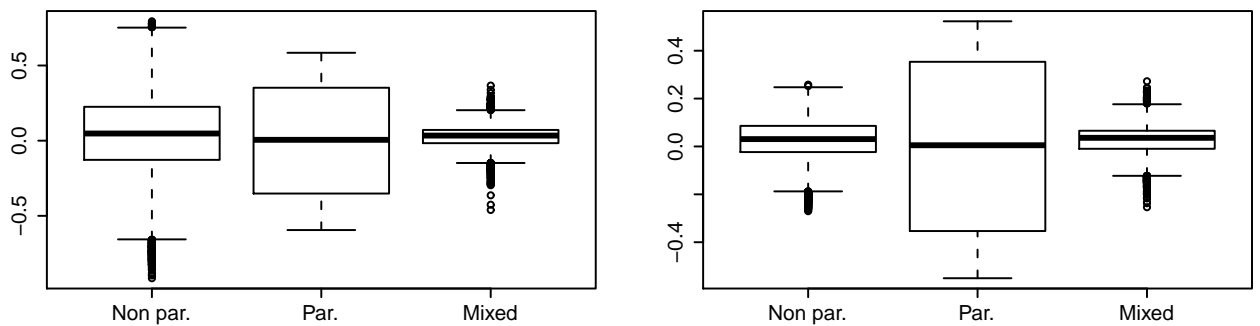

$\theta=0.9, \sigma_{\eta}=0.1$

$\theta=0.1, \sigma_{\eta}=0.1$
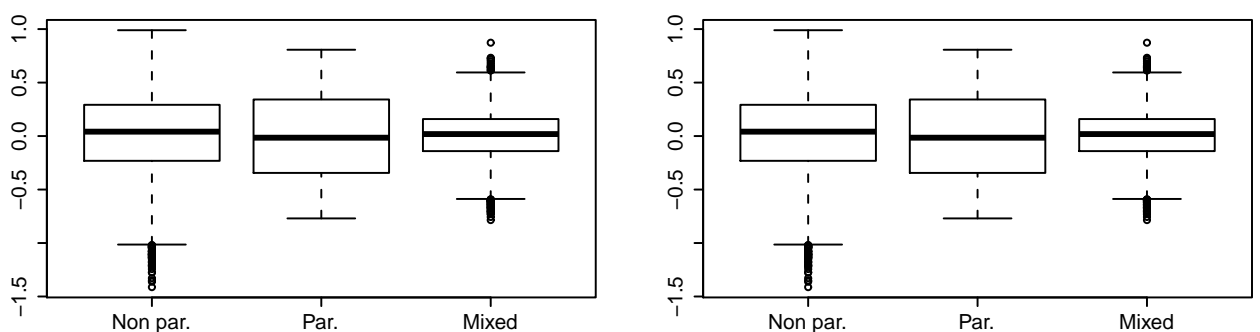

$\theta=0.9, \sigma_{\eta}=0.5$

$\theta=0.1, \sigma_{\eta}=0.5$
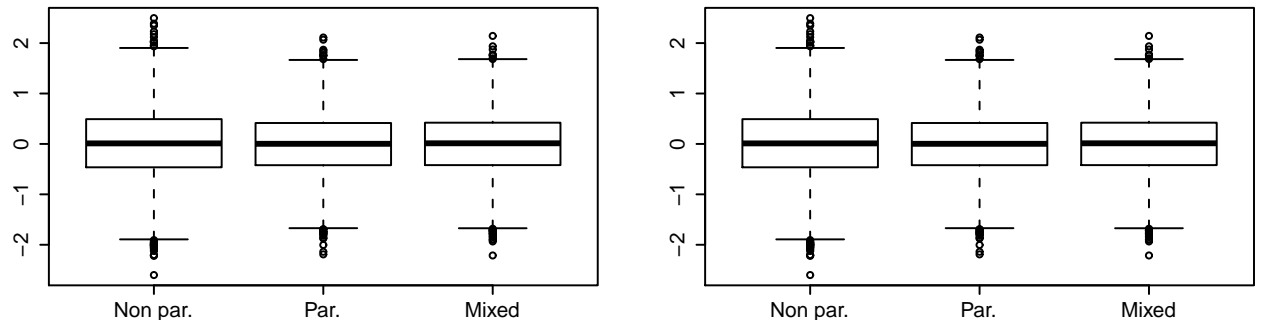

Figure 2: Comparison of the prediction errors of the purely nonparametric, the purely parametric, and the mixed predictors. The simulated process is the $\mathrm{MA}(1) W_{t}=\epsilon_{t-1}+\theta \epsilon_{t-1}$, with $\theta=0.9$ or $\theta=0.1$, where $\left(\epsilon_{t}\right)$ is the weak white noise (4.1) in which $\eta_{t} \sim \mathcal{N}\left(0, \sigma_{\eta}^{2}\right)$ with $\sigma_{\eta}=0.01$ (deterministic proportion $\tau=99.9 \%), \sigma_{\eta}=0.1(\tau=92.5 \%)$ and $\sigma_{\eta}=0.5(\tau=33.3 \%)$.

Table 2: Test that the optimal predictor is a constant and test that the optimal predictor is $\operatorname{ARMA}(1,1): p$-value of the tests of null hypotheses $H_{0}^{W}(d): E\left(W_{t} \mid W_{t-1}, \ldots, W_{t-d}\right) \equiv$ $E W_{t}$ and $H_{0}^{\epsilon}(d): E\left(\epsilon_{t} \mid \epsilon_{t-1} \ldots, \epsilon_{t-d}\right) \equiv 0$, where the $\epsilon_{t}$ 's are approximated by $\operatorname{ARMA}(1,1)$ residuals. In the last four columns, the $p$-values are underlined when they are less than $5 \%$.

\begin{tabular}{lllllllll}
\hline \hline & \multicolumn{3}{c}{$H_{0}^{W}(d)$} & \multicolumn{5}{c}{$H_{0}^{\epsilon}(d)$} \\
BEL & $d=1$ & $d=2$ & $d=3$ & $d=4$ & $d=1$ & $d=2$ & $d=3$ & $d=4$ \\
CAC & 0 & 0 & 0 & 0 & 0.814 & 0.320 & 0.342 & 0.304 \\
DAX & 0.006 & 0 & 0 & 0 & 0.818 & 0.518 & 0.318 & 0.428 \\
FTSE & 0.026 & 0 & 0 & 0 & 0.128 & 0.232 & 0.272 & 0.238 \\
HSI & 0 & 0 & 0 & 0 & $\underline{0.008}$ & 0.062 & 0.242 & 0.274 \\
Nikkei & 0 & 0 & 0 & 0 & 0.062 & $\underline{0.022}$ & $\underline{0.004}$ & $\underline{0.012}$ \\
NSE & 0.004 & 0 & 0 & 0 & 0.160 & $\underline{0.030}$ & 0.090 & 0.116 \\
SMI & 0.016 & 0.002 & 0 & 0 & 0.814 & 0.338 & 0.194 & $\underline{0.046}$ \\
IGBM & 0 & 0 & 0 & 0 & 0.762 & 0.292 & 0.268 & 0.384 \\
SP500 & 0.004 & 0 & 0 & 0 & 0.642 & 0.558 & 0.286 & 0.188 \\
SPTSX & 0.016 & 0 & 0 & 0 & 0.380 & 0.174 & 0.228 & 0.246 \\
SSE & 0 & 0 & 0 & 0 & 0.410 & 0.358 & 0.310 & 0.286 \\
\hline
\end{tabular}


Table 3: RMSE of the ARMA(1,1), nonparametric (NP) and mixed (Mixed) predictions. For each series, the smallest RMSE is underlined.

\begin{tabular}{lrrrrrrrrr}
\hline \hline & \multicolumn{3}{c}{ ARMA(1,1) } & \multicolumn{3}{c}{ Mixed } \\
BEL & $\underline{7.632}$ & 9.165 & 8.901 & 8.592 & 8.856 & 7.742 & 8.087 & 7.694 & 7.647 \\
CAC & 6.177 & 6.764 & 6.767 & 6.746 & 6.635 & 6.205 & 6.275 & $\underline{6.167}$ & 6.209 \\
DAX & $\underline{6.512}$ & 7.133 & 7.077 & 6.973 & 6.949 & 6.516 & 6.586 & 6.598 & 6.540 \\
FTSE & 4.753 & 5.246 & 5.234 & 5.230 & 5.236 & 4.769 & 4.804 & 4.774 & $\underline{4.752}$ \\
HSI & 8.733 & 9.359 & 9.244 & 9.177 & 9.189 & 8.702 & 8.737 & 8.725 & $\underline{8.687}$ \\
Nikkei & 7.338 & 7.973 & 7.975 & 7.961 & 7.975 & $\underline{7.311}$ & 7.318 & 7.352 & 7.347 \\
NSE & 13.505 & 13.693 & 13.545 & 13.654 & 13.540 & 13.497 & 13.453 & 13.434 & $\underline{13.422}$ \\
SMI & $\underline{4.480}$ & 4.974 & 5.025 & 5.017 & 4.948 & 4.502 & 4.533 & 4.513 & 4.489 \\
IGBM & 7.322 & 7.777 & 8.392 & 8.242 & 7.792 & $\underline{7.308}$ & 7.538 & 7.432 & 7.360 \\
SP500 & 5.613 & 6.244 & 6.292 & 6.349 & 6.261 & $\underline{5.612}$ & 5.692 & 5.637 & 5.617 \\
SPTSX & $\underline{6.243}$ & 6.963 & 7.061 & 7.214 & 7.232 & 6.268 & 6.302 & 6.322 & 6.279 \\
SSE & $\underline{8.234}$ & 8.625 & 8.628 & 8.563 & 8.435 & 8.303 & 8.270 & 8.596 & 8.293 \\
\hline
\end{tabular}




\section{Combining nonparametric and optimal linear time series predictions: complementary results}

\section{A Proof of (2.7) and (2.8).}

The dominated convergence theorem entails that, under A1 and A3,

$$
\begin{aligned}
E \hat{f}(x)-f(x) & =\frac{1}{b_{n}^{d}} \int_{\mathbb{R}^{d}} K\left(\frac{x-y}{b_{n}}\right) f(y) d y-f(x) \\
& =\int_{\mathbb{R}^{d}} K(t)\left\{f\left(x-b_{n} t\right)-f(x)\right\} d t \rightarrow 0
\end{aligned}
$$

when $b_{n} \rightarrow 0$.

By stationarity, we have

$$
\operatorname{Var} \hat{f}(x)=\frac{1}{n b_{n}^{2 d}} \sum_{h=-n+1}^{n-1}\left(\frac{n-|h|}{n}\right) \operatorname{Cov}\left\{K\left(\frac{x-X_{t}}{b_{n}}\right), K\left(\frac{x-X_{t-|h|}}{b_{n}}\right)\right\} .
$$

Davydov's inequality (1968) entails

$$
\begin{aligned}
& \left|\operatorname{Cov}\left\{K\left(\frac{x-X_{t}}{b_{n}}\right), K\left(\frac{x-X_{t-|h|}}{b_{n}}\right)\right\}\right| \\
\leq & C\left\|K\left(\frac{x-X_{t}}{b_{n}}\right)\right\|_{2+\nu}^{2}\left\{\alpha_{X}(|h|)\right\}^{\frac{\nu}{2+\nu}} .
\end{aligned}
$$

Note that, in view of the Lipschitz condition $\mathbf{A 1}$, the density $K$ is (uniformly) continuous. Thus it is bounded and satisfies $\int K^{2+\nu}(t) d t<\infty$. Thus, using A1-A3 and Lemma 2.1, $\operatorname{Var} \hat{f}(x) \rightarrow 0$ as $n b_{n}^{d\left(1+\frac{\nu}{2+\nu}\right)} \rightarrow \infty$. The first part of (2.7) is shown.

Now we will show that $\hat{\varphi}(x)=\varphi(x)+o_{P}(1)$. The second part of (2.7) will follow, using the Slutsky lemma. With the arguments used to handle $\operatorname{Var} \hat{f}(x)$ we obtain

$$
\begin{aligned}
& \operatorname{Var} \hat{\varphi}(x) \leq \frac{C}{n b_{n}^{2 d}}\left\|g\left(Y_{t}\right) K\left(\frac{x-X_{t}}{b_{n}}\right)\right\|_{2+\nu}^{2} \\
= & \frac{C}{n b_{n}^{2 d}}\left\{b_{n}^{d} \int|g(y)|^{2+\nu} K^{2+\nu}(t) f_{Z}\left(y, x-b_{n} t\right) d y d t\right\}^{\frac{2}{2+\nu}} \\
\leq & \frac{C}{n b_{n}^{d\left(1+\frac{\nu}{2+\nu}\right)}}\left\{\sup _{u \in \mathbb{R}^{d}} \int_{\mathbb{R}^{d_{0}}}|g(y)|^{2+\nu} f_{Z}(y, u) d y \int_{\mathbb{R}^{d}} K^{2+\nu}(t) d t\right\}^{\frac{2}{2+\nu}}=o(1)
\end{aligned}
$$

under A1-A4. Now note that

$$
\begin{aligned}
E \hat{\varphi}(x) & =b_{n}^{-d} \int_{\mathbb{R}^{d}}\left\{\int_{\mathbb{R}^{d_{0}}} g(y) \frac{f_{Z_{t}}(y, u)}{f(u)} d y\right\} K\left(\frac{x-u}{b_{n}}\right) f(u) d u \\
& =b_{n}^{-d} \int_{\mathbb{R}^{d}} r(u) f(u) K\left(\frac{x-u}{b_{n}}\right) d u=\int_{\mathbb{R}^{d}} K(t) \varphi\left(x-b_{n} t\right) d t \\
& =\int_{\mathbb{R}^{d}} K(t)\left\{\varphi(x)-b_{n} t^{\prime} \frac{\partial \varphi}{\partial x}(x)+\frac{b_{n}^{2}}{2} t^{\prime} \frac{\partial^{2} \varphi}{\partial x \partial x^{\prime}}\left(x^{*}\right) t\right\} d t,
\end{aligned}
$$


where $x^{*}$ is between $x$ and $x-b_{n} t$, which shows that the bias of $\hat{\varphi}(x)$ tends to zero. The proof of (2.7) is complete.

A Taylor expansion yields

$$
f\left(x-b_{n} t\right)=f(x)-b_{n} t^{\prime} \frac{\partial f}{\partial x}(x)+\frac{b_{n}^{2}}{2} t^{\prime} \frac{\partial^{2} f}{\partial x \partial x^{\prime}}\left(x^{*}\right) t,
$$

where $x^{*}$ is between $x$ and $x-b_{n} t$. Using (A.1), A1 and A3, we obtain

$$
E \hat{f}(x)-f(x)=O\left(b_{n}^{2}\right) \quad \text { and } \quad \operatorname{Var} \hat{f}(x)=O\left(n^{-1} b_{n}^{-d\left(1+\frac{\nu}{2+\nu}\right)}\right) .
$$

Thus

$$
E\{\hat{f}(x)-f(x)\}^{2}=O\left(b_{n}^{4}+n^{-1} b_{n}^{-d\left(1+\frac{\nu}{2+\nu}\right)}\right)
$$

is asymptotically minimal for $b_{n}=O\left(n^{-1 /\{4+d+d \nu /(2+\nu)\}}\right)$. We obtain the result for the regression estimator by the same arguments. We deduce that (2.8) holds.

\section{B Proof of (2.16).}

Write

$$
\sqrt{n b_{n}^{d}}\{\hat{r}(x)-r(x)\}=\frac{\sqrt{n b_{n}^{d}}\{\hat{\varphi}(x)-\varphi(x)\}}{\hat{f}(x)}-\varphi(x) \frac{\sqrt{n b_{n}^{d}}\{\hat{f}(x)-f(x)\}}{\hat{f}(x) f(x)},
$$

and let $H_{n}(x)=\left(\sqrt{n b_{n}^{d}}\{\hat{f}(x)-f(x)\}, \sqrt{n b_{n}^{d}}\{\hat{\varphi}(x)-\varphi(x)\}\right)^{\prime}$. For any $c=\left(c_{1}, c_{2}\right)^{\prime} \in \mathbb{R}^{2}$, letting $g_{c}\left(Y_{t}\right)=c_{1}+c_{2} g\left(Y_{t}\right)$, we have

$$
\begin{aligned}
c^{\prime} H_{n}(x)= & \frac{1}{\sqrt{n b_{n}^{d}}} \sum_{t=1}^{n} g_{c}\left(Y_{t}\right) K\left(\frac{x-X_{t}}{b_{n}}\right) \\
& -\sqrt{n b_{n}^{d}}\left\{c_{1} f(x)+c_{2} \varphi(x)\right\} \\
= & \frac{1}{\sqrt{n}} S_{n}+R_{n},
\end{aligned}
$$

where $S_{n}=\sum_{t=1}^{n} x_{n, t}$,

$$
x_{n, t}=\frac{1}{b_{n}^{d / 2}}\left\{g_{c}\left(Y_{t}\right) K\left(\frac{x-X_{t}}{b_{n}}\right)-E\left[g_{c}\left(Y_{t}\right) K\left(\frac{x-X_{t}}{b_{n}}\right)\right]\right\},
$$

and

$$
R_{n}=\frac{1}{\sqrt{n b_{n}^{d}}} \sum_{t=1}^{n}\left\{E\left[g_{c}\left(Y_{t}\right) K\left(\frac{x-X_{t}}{b_{n}}\right)\right]-b_{n}^{d}\left\{c_{1} f(x)+c_{2} \varphi(x)\right\}\right\} .
$$

To establish the asymptotic normality of $c^{\prime} H_{n}(x)$, for $c \neq 0$, we will verify the conditions for a Central Limit Theorem for triangular arrays. Notice that the strong mixing coefficients $\alpha_{n}(h)$ of the process $\left(x_{n, t}\right)_{t \in \mathbb{Z}}$ are such that $\alpha_{n}(h) \leq \alpha_{Z}(h)$. By stationarity, we have

$$
\frac{1}{n} \operatorname{Var} S_{n}=\sum_{h=-n+1}^{n-1}\left(\frac{n-|h|}{n}\right) \operatorname{Cov}\left(x_{n, t}, x_{n, t-|h|}\right) .
$$


Davydov's inequality (1968) and a direct extension of Lemma 2.1 entail

$$
\begin{aligned}
\left|\operatorname{Cov}\left(x_{n, t}, x_{n, t-|h|}\right)\right| & \leq \frac{C}{b_{n}^{d}}\left\|g_{c}\left(Y_{t}\right) K\left(\frac{x-X_{t}}{b_{n}}\right)\right\|_{2+\nu}^{2}\left\{\alpha_{Z}(|h|)\right\}^{\frac{\nu}{2+\nu}} \\
& \leq C b_{n}^{\frac{-d \nu}{2+\nu}}\left\{\alpha_{Z}(|h|)\right\}^{\frac{\nu}{2+\nu}} .
\end{aligned}
$$

Now we will show that

$$
\left|\operatorname{Cov}\left(x_{n, t}, x_{n, t-|h|}\right)\right| \leq\left\{\begin{array}{l}
C \text { if } h=0 \\
C b_{n}^{d} \quad \text { if }|h|>0 .
\end{array}\right.
$$

First considering the case $h=0$, we have

$$
\begin{aligned}
\operatorname{Var}\left(x_{n, t}\right) & \leq \frac{1}{b_{n}^{d}} \int g_{c}^{2}(y) K^{2}\left(\frac{x-u}{b_{n}}\right) f_{Z}(y, u) d y d u \\
& =\int g_{c}^{2}(y) K^{2}(v) f_{Z}\left(y, x-v b_{n}\right) d y d v \\
& \leq C \int g_{c}^{2}(y) K^{2}(v) f_{Y}(y) d y d v<+\infty
\end{aligned}
$$

where the second inequality follows from $\mathbf{A} 3$ ' and the last one from $\mathbf{A} 1$ and $\mathbf{A} 3$. This establishes (B.5) in the case $h=0$. Now we consider the case $h>0$ which will be sufficient to conclude. Note that

$$
\begin{aligned}
E g_{c}\left(Y_{t}\right) K\left(\frac{x-X_{t}}{b_{n}}\right)= & c_{1} b_{n}^{d} \int K(v) f\left(x-v b_{n}\right) d v \\
& +c_{2} b_{n}^{d} \int g(y) K(v) f_{Z}\left(y, x-v b_{n}\right) d y d v \\
= & c_{1} b_{n}^{d} f(x)+c_{2} b_{n}^{d} \varphi(x)+o\left(b_{n}^{d}\right) .
\end{aligned}
$$

It follows that

$$
\begin{aligned}
& \left|\operatorname{Cov}\left(x_{n, t}, x_{n, t-h}\right)\right| \\
\leq & \frac{1}{b_{n}^{d}} \int\left|g_{c}\left(y_{1}\right)\right| K\left(\frac{x-u_{1}}{b_{n}}\right)\left|g_{c}\left(y_{2}\right)\right| K\left(\frac{x-u_{2}}{b_{n}}\right) f_{Z_{h}, Z_{0}}\left(y_{1}, u_{1}, y_{2}, u_{2}\right) d y_{1} d u_{1} d y_{2} d u_{2}+C b_{n}^{d} \\
\leq & C b_{n}^{d}\left\{\int\left|g_{c}\left(y_{1}\right)\right| K\left(v_{1}\right)\left|g_{c}\left(y_{2}\right)\right| K\left(v_{2}\right) f_{Z_{h}, Z_{0}}\left(y_{1}, x-v_{1} b_{n}, y_{2}, x-v_{2} b_{n}\right) d y_{1} d v_{1} d y_{2} d v_{2}+1\right\} \\
\leq & C b_{n}^{d}\left\{\int\left|g_{c}\left(y_{1}\right)\right| K\left(v_{1}\right)\left|g_{c}\left(y_{2}\right)\right| K\left(v_{2}\right) f_{Y_{h}, Y_{0}}\left(y_{1}, y_{2}\right) d y_{1} d v_{1} d y_{2} d v_{2}+1\right\} \leq C b_{n}^{d},
\end{aligned}
$$

using A3' and the Schwarz inequality. Now we consider a truncation of the right-hand side of (B.3). Let $\varsigma=d /(4+d)$. We have, by (B.5) and A4'

$$
\sum_{|h|=1}^{\left[n^{\varsigma}\right]}\left(\frac{n-|h|}{n}\right) \operatorname{Cov}\left(x_{n, t}, x_{n, t-|h|}\right) \leq C b_{n}^{d} n^{\varsigma}=o(1) .
$$

Moreover, by (B.4) and A4'

$$
\begin{aligned}
\sum_{|h|=\left[n^{\varsigma}\right]+1}^{n}\left(\frac{n-|h|}{n}\right) \operatorname{Cov}\left(x_{n, t}, x_{n, t-|h|}\right) & \leq C b_{n}^{\frac{-d \nu}{2+\nu}} \sum_{|h|>\left[n^{\varsigma}\right]}\left\{\alpha_{Z}(|h|)\right\}^{\frac{\nu}{2+\nu}} \\
& \leq C b_{n}^{\frac{-d \nu}{2+\nu}} n^{-\varrho \varsigma}=o(1) .
\end{aligned}
$$


The last inequality follows from $\left\{\alpha_{Z}(|h|)\right\}^{\frac{\nu}{2+\nu}}=O\left(h^{-(\varrho+1)}\right)$, which is a consequence of A2', and from a standard comparison with an integral. It follows that

$$
\lim _{n \rightarrow \infty} \frac{1}{n} \operatorname{Var} S_{n}=\lim _{n \rightarrow \infty} \operatorname{Var} x_{n, t}=E\left(g_{c}^{2}\left(Y_{t}\right) \mid X_{t}=x\right) f(x) \int_{\mathbb{R}^{d}} K^{2}(u) d u
$$

where the second equality is a consequence of (B.6). Thus, applying a CLT for triangular sequences of mixing sequences (see e.g. the book by Davidson (1994) and the references therein),

$$
n^{-1 / 2} S_{n} \stackrel{d}{\rightarrow} \mathcal{N}\left(0,\left\{c_{1}^{2} f(x)+2 c_{1} c_{2} \varphi_{1}(x)+c_{2}^{2} \varphi_{2}(x)\right\} \int_{\mathbb{R}^{d}} K^{2}(u) d u\right) .
$$

Now we have, in view of (A.2) and a similar expression for the difference $E \hat{\varphi}(x)-\varphi(x)$, by $\mathrm{A} 4$,

$$
R_{n}=c_{1} \sqrt{n b_{n}^{d}}\{E \hat{f}(x)-f(x)\}+c_{2} \sqrt{n b_{n}^{d}}\{E \hat{\varphi}(x)-\varphi(x)\}=o(1) .
$$

Thus, (B.2) entails that $c^{\prime} H_{n}(x)$ has the same asymptotic distribution as $n^{-1 / 2} S_{n}$. Finally, in view of (B.1) and (2.7) $\sqrt{n b_{n}^{d}}\{\hat{r}(x)-r(x)\}$ has the same asymptotic distribution as $c^{\prime} H_{n}(x)$ with $c=(-r(x) / f(x), 1 / f(x))^{\prime}$, which completes the proof of (2.16).

\section{Simulations of the model of Section 4.1.}

Figure 3 plots simulations of the noise $\left(\epsilon_{t}\right)$ and of the weak $\mathrm{MA}(1)$ process $\left(W_{t}\right)$. The empirical autocorrelation functions are in accordance with the theoretical second-order structure of the two simulated processes. In particular, on the basis of the correlogramm, a practitioner would certainly select the $\mathrm{MA}(1)$ as a plausible model. One can observe that the distribution of the noise is symmetric, whereas that of the MA(1) is clearly asymmetric. Such an asymmetry is not possible for a MA(1) process with an iid symmetric noise. The simulated trajectories displayed in Figure 3 correspond to a noise with a deterministic proportion $\tau=92.6 \%$. The asymmetry is of course less marked when the deterministic proportion $\tau$ is smaller or when the MA(1) parameter $\theta$ is close to 0 .

\section{Complements on the stock index data of Section 4.2.}

Figure 6 displays the autocorrelation functions of the returns. In this figure, the dotted lines $\pm 1.96 / \sqrt{n}$ define the standard significance band in which the autocorrelations of an iid noise should stay with asymptotic probability $95 \%$. These significance bands, obtained from an application of the well-known standard Bartlett's formula, are not valid when the observations are uncorrelated but not independent, as it is the case for GARCH processes, and more generally for weak white noises. Significant bands obtained from the generalized Bartlett's formula recently proposed by Francq and Zakoïan (2009), are given in full lines. Given that most of the autocorrelations fall into the generalized Bartlett's bands, it is reasonable to consider the returns as weak white noises. This is in agreement with the standard economic theory which asserts that such stock returns should be martingale differences. In view of Figure 7, displaying the autocorrelation functions of the squares of the returns, it is however clear that the squares of the returns are correlated. 
Simulation of the noise

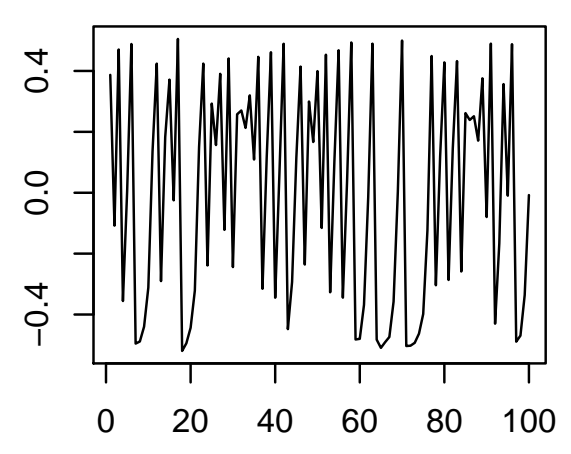

Simulation of the MA(1)

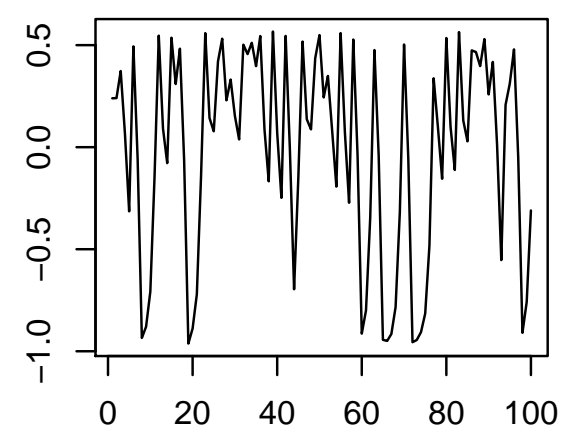

ACF of the noise

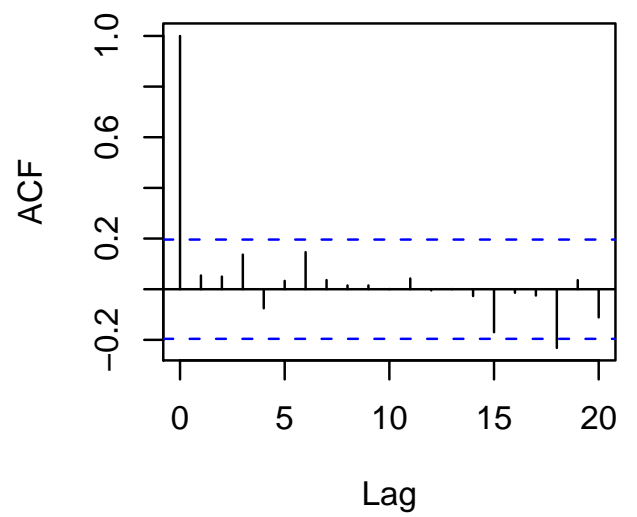

ACF of the MA(1)

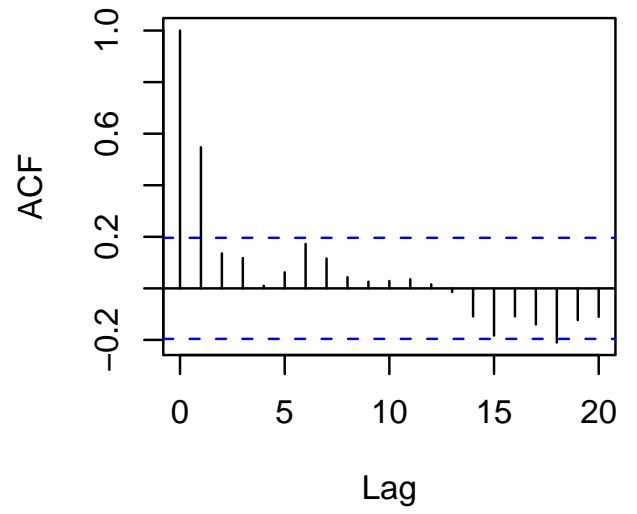

Figure 3: A simulation of the weak white noise $\epsilon_{t}$ defined by (4.1) with $\eta_{t} \sim \mathcal{N}\left(0, \sigma_{\eta}^{2}\right)$ and $\sigma_{\eta}=0.1$, and a simulation of the $\mathrm{MA}(1)$ process $W_{t}=\epsilon_{t-1}+0.9 \epsilon_{t-1}$. The right panels display the autocorrelation functions of the two simulations. 

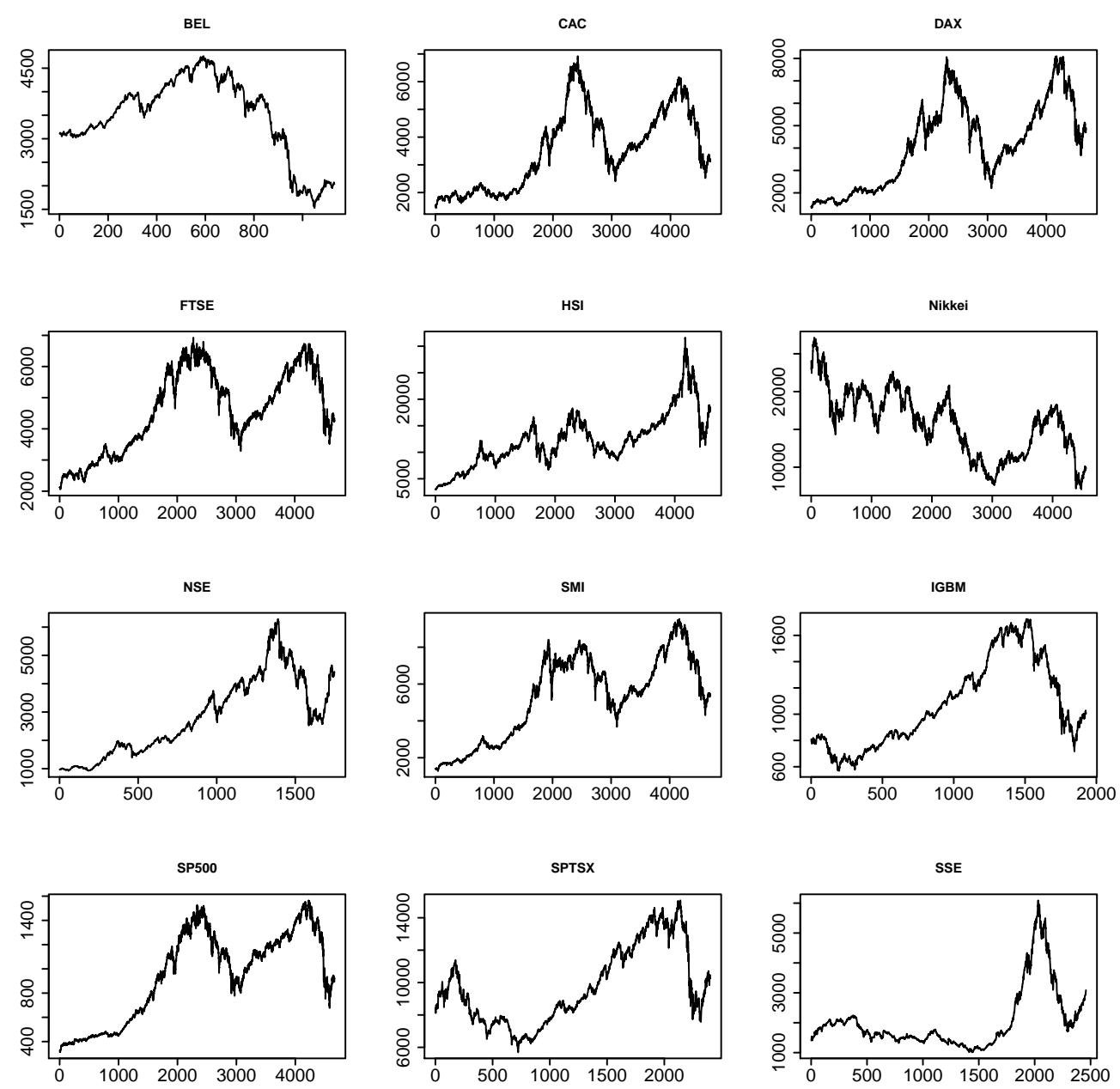

Figure 4: World financial indices between January 2, 1991 and July 3, 2009. 

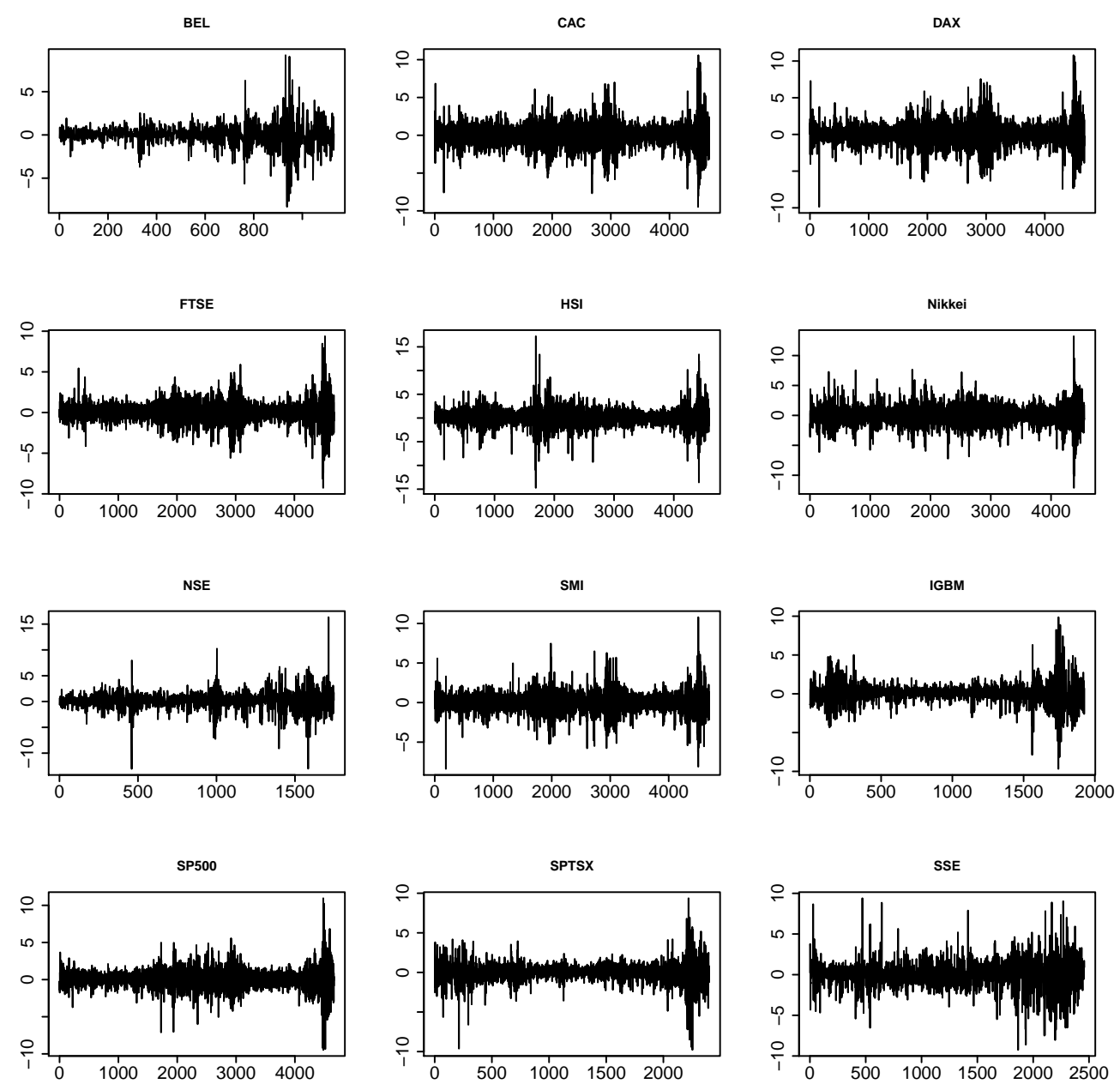

Figure 5: Returns of the indices of Figure 4. 

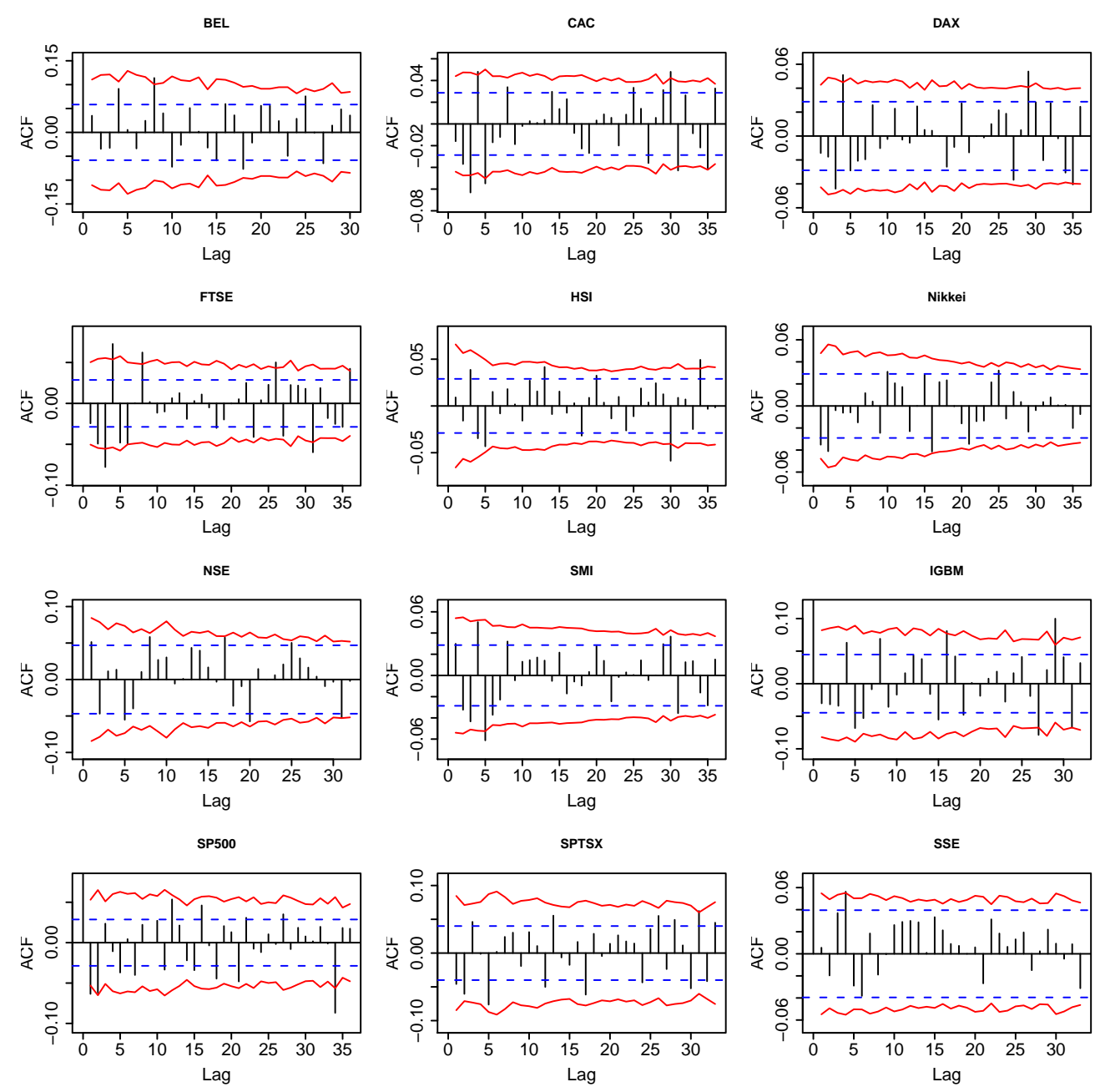

Figure 6: Autocorrelation functions of the series of the returns.

Figures 8-10 display, for each index, the Nadaraya-Watson estimator $\hat{r}(x)$ of the regression $r(x)=E\left(W_{t} \mid W_{t-k}=x\right)$ for the lag $k=1$ and $k=7$. The dotted lines are $95 \%$ confidence bands for the regression, deduced from (2.16). It is interesting to note that the general allure of all these regressions is that of increasing functions. This is in accordance with the usual finding of strong positive correlations for the squared returns. 

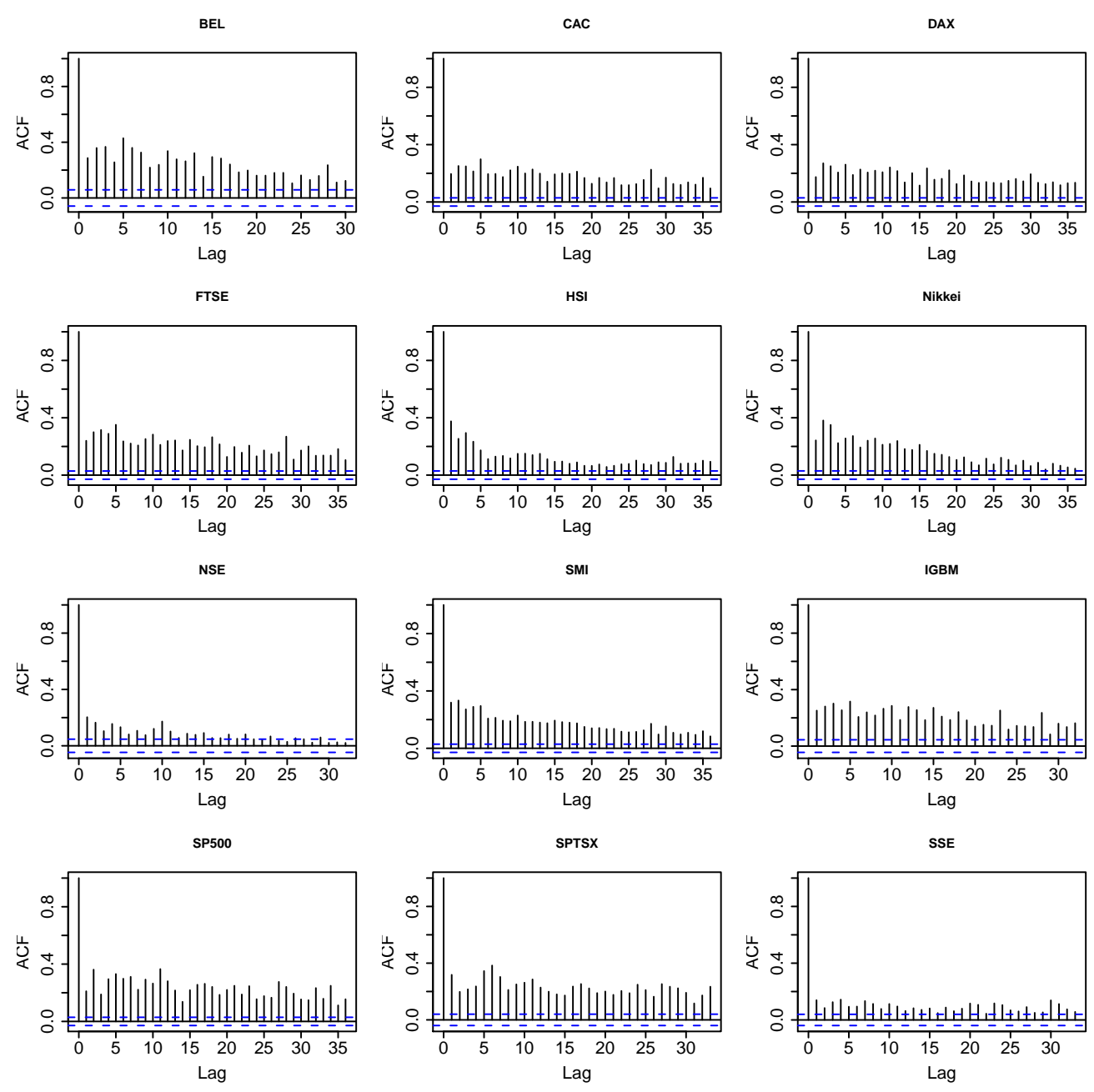

Figure 7: Autocorrelation functions of the squares of the returns. 
BEL
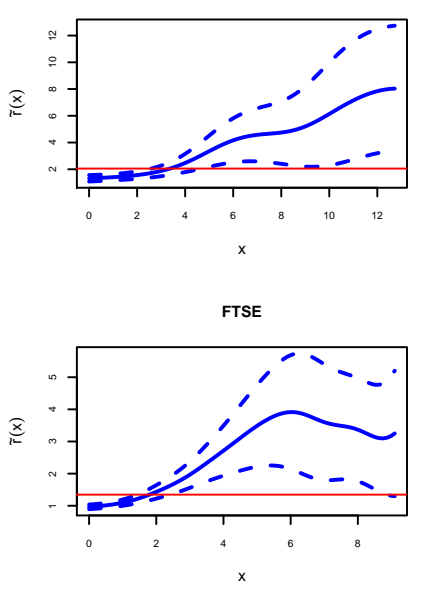

NSE

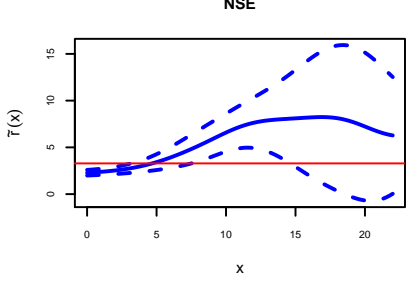

SP500

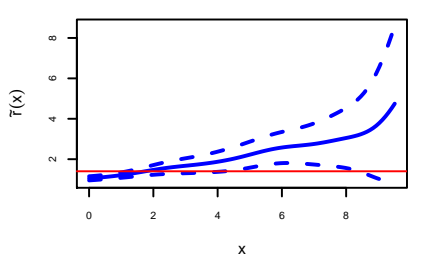

CAC
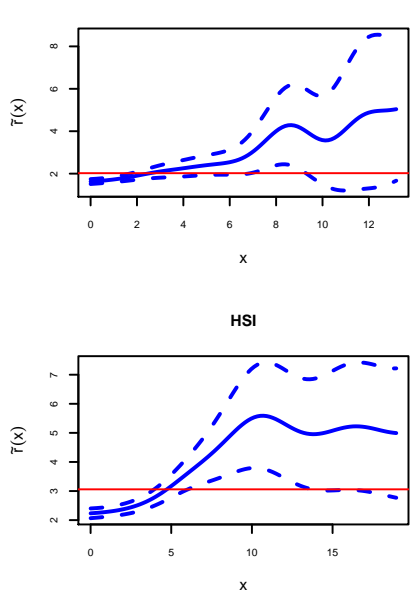

SMI

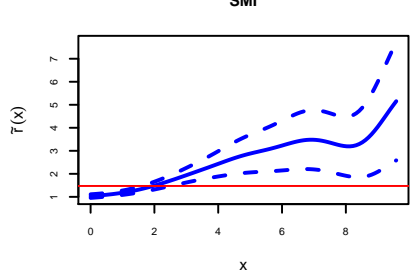

SPTSX

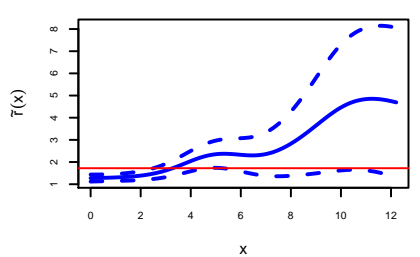

DAX

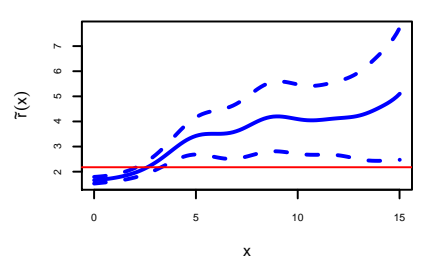

Nikkei

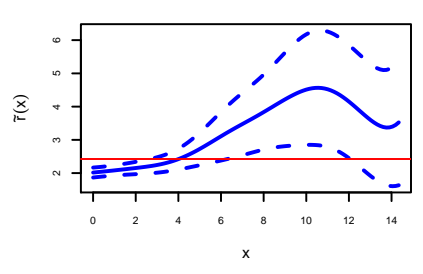

IGBM

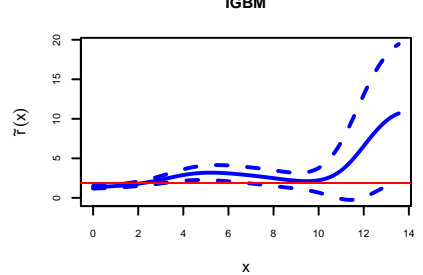

SSE

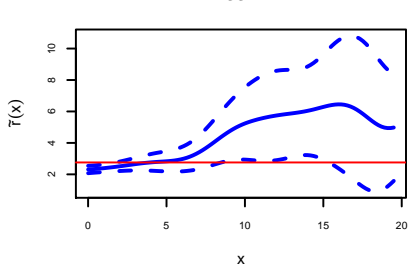

Figure 8: Nadaraya-Watson estimator $\hat{r}(x)$ of the regression $r(x)=E\left(W_{t} \mid W_{t-1}=x\right)$, where $W_{t}$ denotes the squares of the returns. 

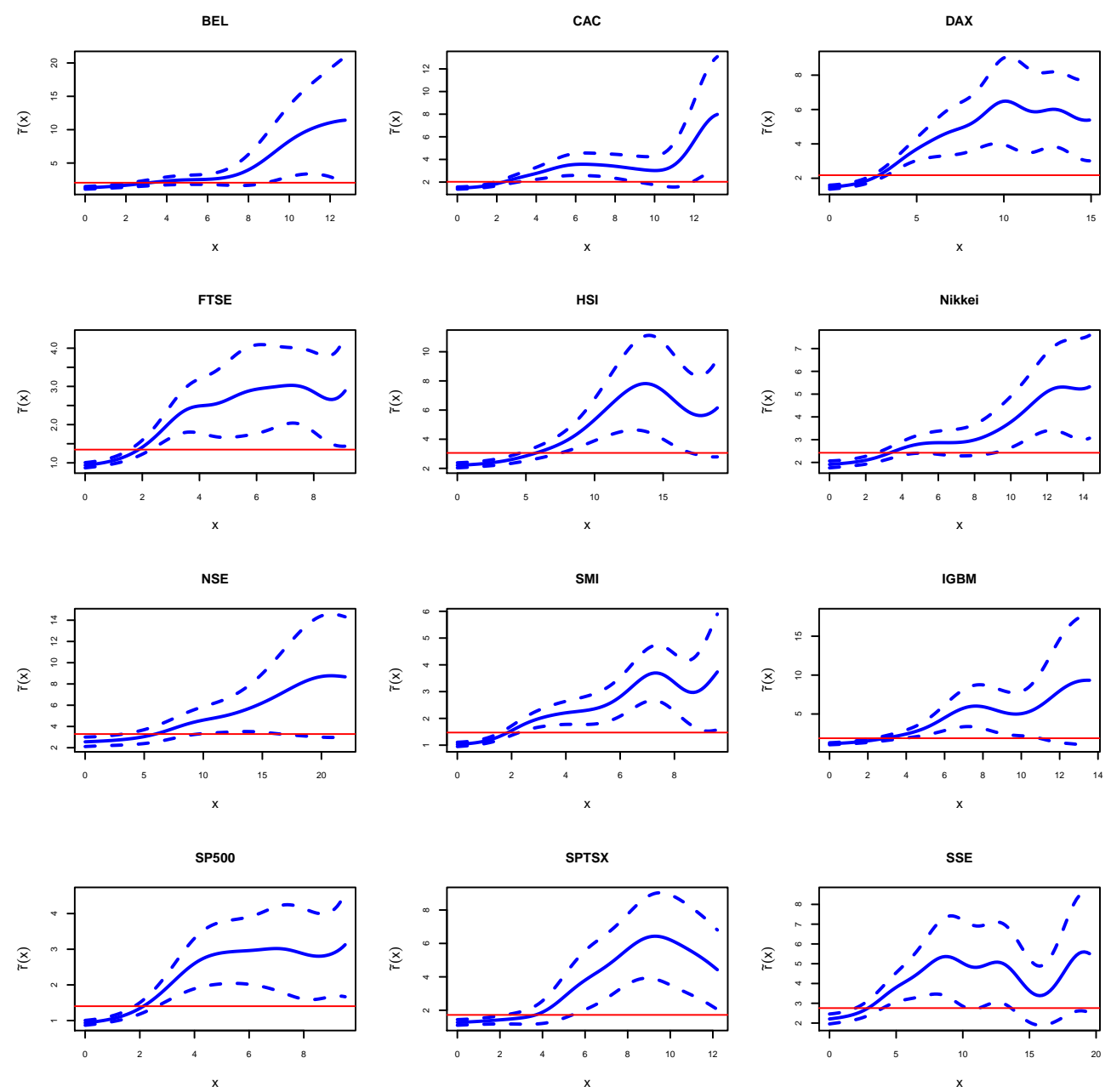

Figure 9: As Figure 8, but for the lag 2 regression $r(x)=E\left(W_{t} \mid W_{t-2}=x\right)$. 
BEL

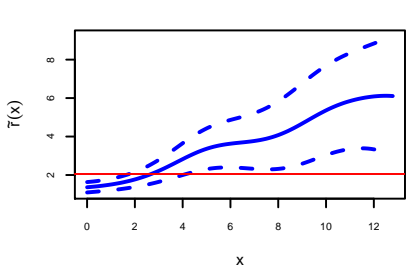

FTSE
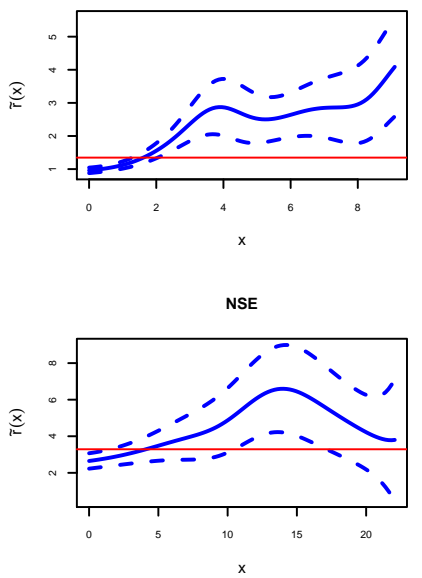

SP500

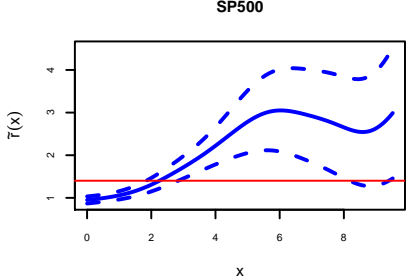

CAC

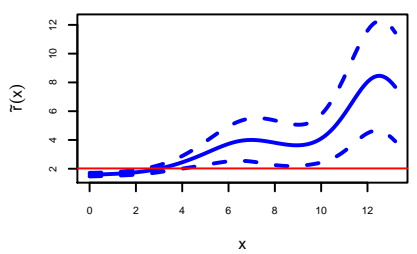

HSI

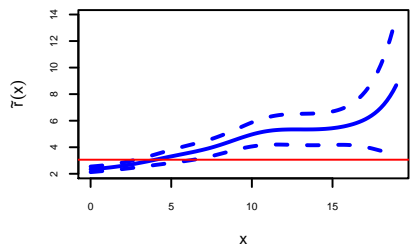

SMI

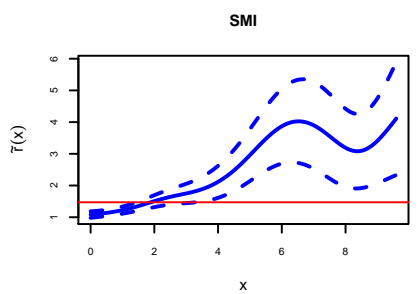

SPTSX

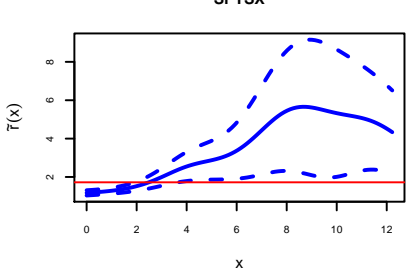

DAX

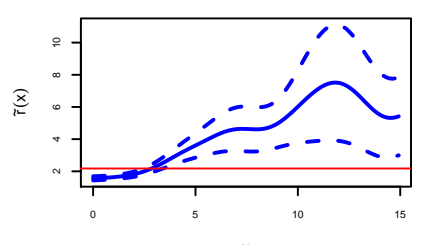

Nikkei
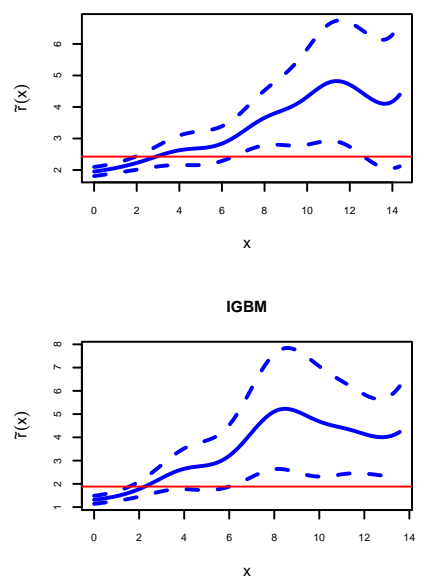

SSE

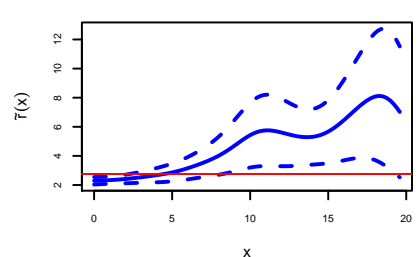

Figure 10: As Figure 8, but for the higher lag regression $r(x)=E\left(W_{t} \mid W_{t-7}=x\right)$. 
BEL

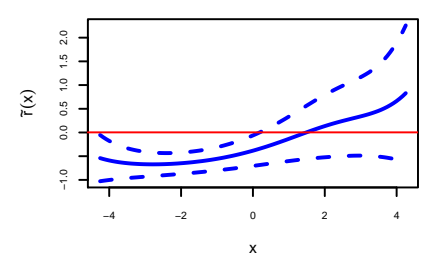

FTSE

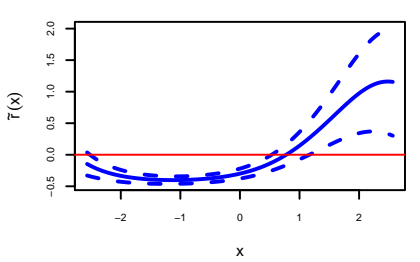

NSE

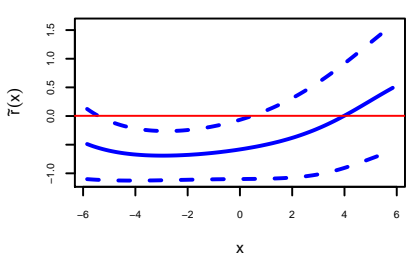

SP500

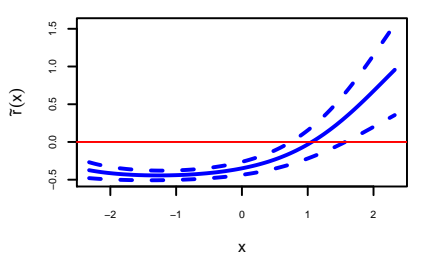

CAC

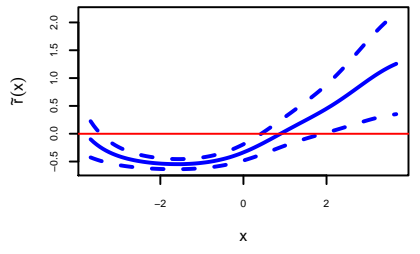

HSI

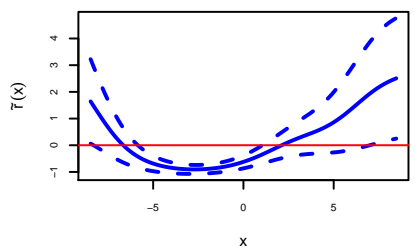

SMI

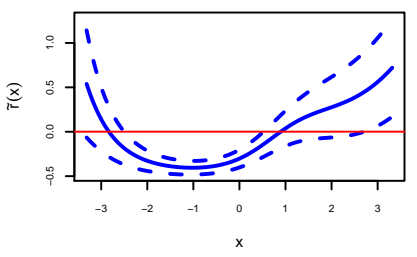

SPTSX

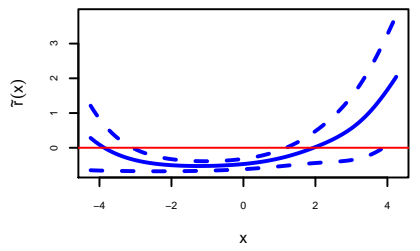

DAX

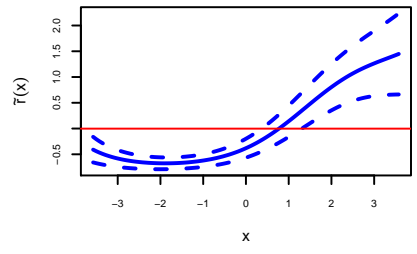

Nikkei

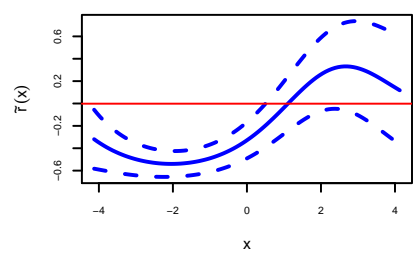

IGBM

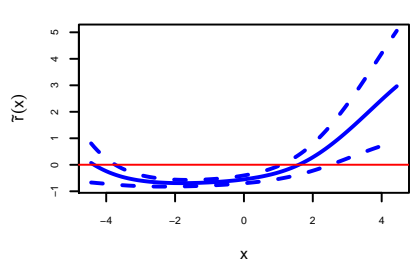

SSE

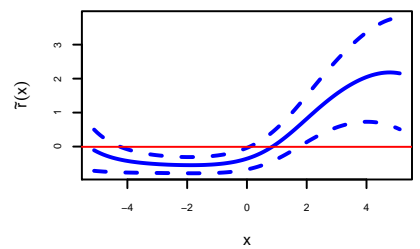

Figure 11: Non parametric estimator of $r(x)=E\left(\epsilon_{t} \mid \epsilon_{t-1}=x\right)$ where $\epsilon_{t}$ is the MA(1) error term.

The first columns of Table 4 give the $p$-values of the KNY test that the regression of $W_{t}$ on $W_{t-1}, \ldots, W_{t-d}$ is constant. This assumption is clearly rejected, confirming the visual aspect of the regressions displayed in Figures 8-10, and also the strong autocorrelations of $\left(W_{t}\right)$ displayed in Figure 7 . The last columns of Table 4 concern the test described in Section 3.5 that the best predictor is the MA(1) model. Since the assumption is clearly rejected, the MA(1) predictor is likely to be beaten by a purely non parametric predictor or by a mixed-predictor. Figure 11 confirms the output of the tests, since numerous regressions have a "smile" form which leads to predict a positive $\epsilon_{t}$ (and thus a larger volatility) when the innovation $\epsilon_{t-1}$ is far from zero. The asymmetry of the smile indicates that the volatility increases more when $W_{t-1}$ is higher than when it is lower than expected. 
Table 4: Test that the optimal predictor is a constant and test that the optimal predictor is MA(1): $p$-value of the tests of null hypotheses $H_{0}^{W}(d): E\left(W_{t} \mid W_{t-1}, \ldots, W_{t-d}\right) \equiv E W_{t}$ and $H_{0}^{\epsilon}(d): E\left(\epsilon_{t} \mid \epsilon_{t-1} \ldots, \epsilon_{t-d}\right) \equiv 0$, where the $\epsilon_{t}$ 's are approximated by MA(1) residuals.

\begin{tabular}{|c|c|c|c|c|c|c|c|c|}
\hline & \multicolumn{4}{|c|}{$H_{0}^{W}(d)$} & \multicolumn{4}{|c|}{$H_{0}^{\epsilon}(d)$} \\
\hline & $d=1$ & $d=2$ & $d=3$ & $d=4$ & $d=1$ & $d=2$ & $d=3$ & $d=4$ \\
\hline BEL & 0 & 0 & 0 & 0 & 0.010 & 0 & 0 & 0 \\
\hline $\mathrm{CAC}$ & 0.006 & 0 & 0 & 0 & 0.084 & 0 & 0 & 0 \\
\hline DAX & 0.026 & 0 & 0 & 0 & 0.370 & 0 & 0 & 0 \\
\hline FTSE & 0 & 0 & 0 & 0 & 0.070 & 0 & 0 & 0 \\
\hline HSI & 0 & 0 & 0 & 0 & 0 & 0 & 0 & 0 \\
\hline Nikkei & 0.004 & 0 & 0 & 0 & 0.002 & 0 & 0 & 0 \\
\hline NSE & 0.016 & 0.002 & 0 & 0 & 0.118 & 0.014 & 0.002 & 0 \\
\hline SMI & 0 & 0 & 0 & 0 & 0.044 & 0 & 0 & 0 \\
\hline IGBM & 0.004 & 0 & 0 & 0 & 0.088 & 0 & 0 & 0 \\
\hline SP500 & 0.016 & 0 & 0 & 0 & 0.008 & 0 & 0 & 0 \\
\hline SPTSX & 0 & 0 & 0 & 0 & 0.006 & 0 & 0 & 0 \\
\hline SSE & 0.178 & 0.006 & 0 & 0 & 0.998 & 0.128 & 0 & 0 \\
\hline
\end{tabular}

\section{E $\quad R$ code}

This section contains programs written in the R language (see http://cran.r-project.org/). We begin with three auxiliary routines: the function $\mathrm{K}($ ) for the multivariate gaussian kernel, the function $\mathrm{rn}()$ for the Nadaraya-Watson regressor of $\mathrm{Y}$ on the columns of $\mathrm{X}$ at the point $\mathrm{x}$ with the bandwidth $\mathrm{h}$, and the function bandwidth. $\mathrm{x}()$ for an approximation of the local optimal bandwidth at the point $\mathrm{x}$ (as described in Bosq, 1996, Chapter 2).

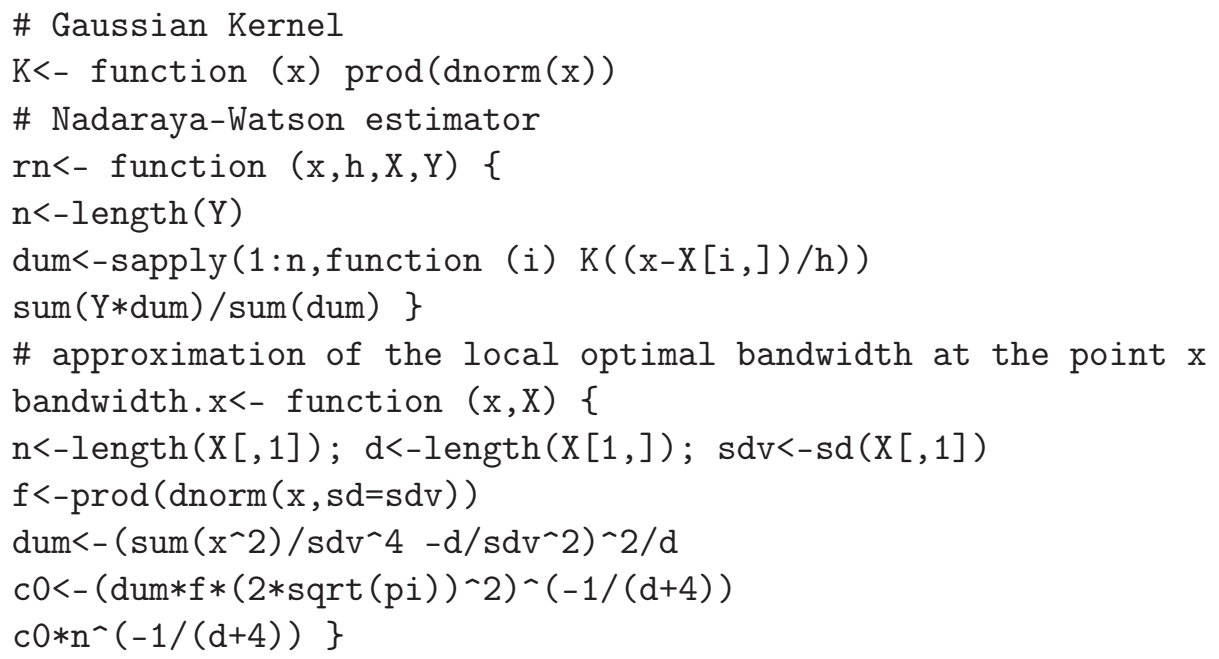

Given a time series $W[1: n]$, the function $\operatorname{prevNP}()$ uses the function $r n()$ to compute the non parametric Nadaraya-Watson predictor of $\mathrm{W}[\mathrm{n}+1]$ as function of $d$ past values.

\# nonparametric prediction of $W[n+1]$ as a function of $x=(W[n], \ldots, W[n-d+1])$ prevNP<- function (W,d) \{

$\mathrm{n}<-$ length $(\mathrm{W}) ; \mathrm{x}<-\mathrm{W}[\mathrm{n}:(\mathrm{n}-\mathrm{d}+1)]$ 
$\mathrm{X}<-$ matrix $($ nrow $=(\mathrm{n}-\mathrm{d}), \mathrm{ncol}=\mathrm{d})$

for $(j$ in $(1: d)) x[, j]<-W[(d+1-j):(n-j-$ delay $)]$

$\mathrm{h}<-$ bandwidth. $\mathrm{x}(\mathrm{x}, \mathrm{X})$

prev< $<-r n(x, h, X, W[(d+1+$ delay $): n])\}$

The function prevPara.arma11() uses the function arma() of the library tseries to compute the $\operatorname{ARMA}(1,1)$ prediction of $\mathrm{W}[\mathrm{n}+1]$.

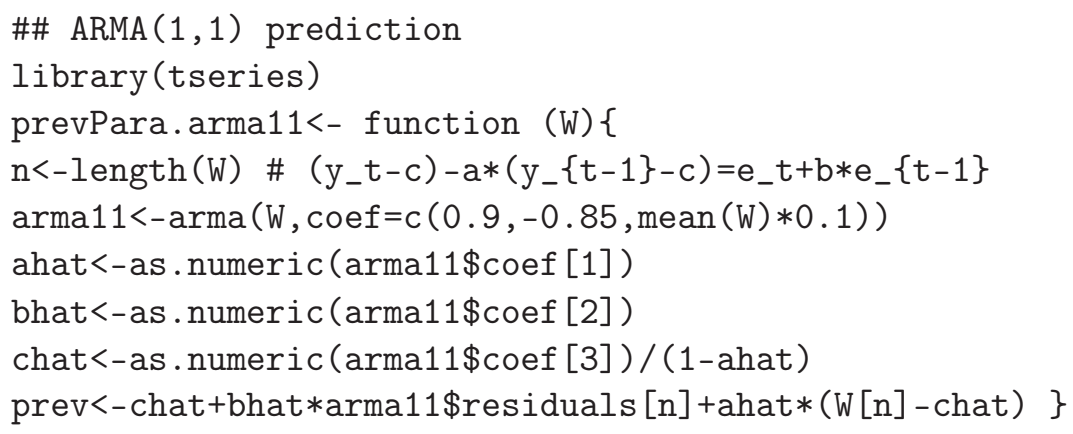

The function prevMixte.arma11() provides a mixed prediction, sum of an $\operatorname{ARMA}(1,1)$ prediction of $W[n+1]$ and of a non parametric prediction of res $[n+1]$.

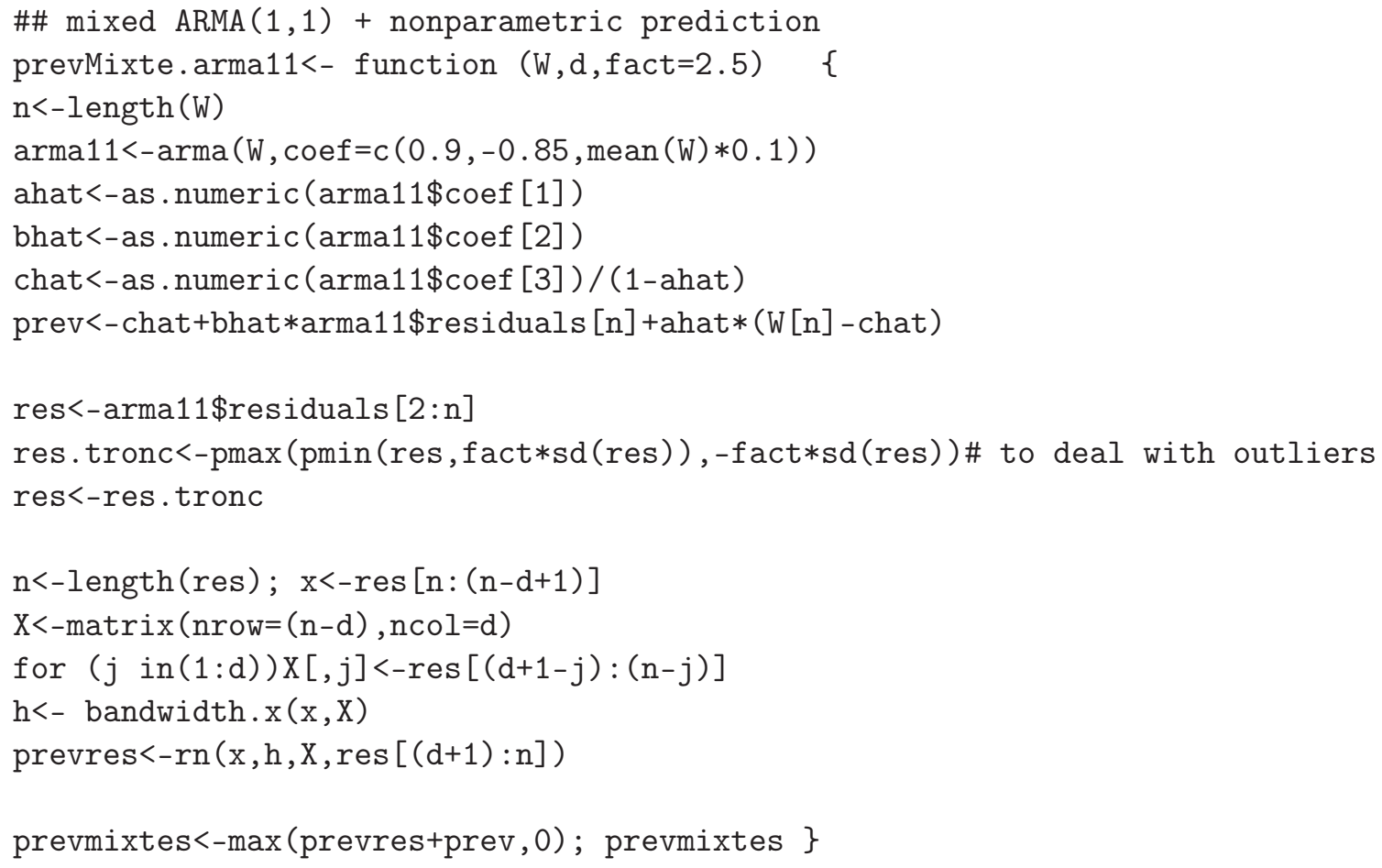




\section{References for the supplementary part}

Davidson, J. (1994) Stochastic Limit Theory. Oxford University Press, New-York.

Davydov, Y.A. (1968) Convergence of Distributions Generated by Stationary Stochastic Processes. Theory of Probability and its Applications 13, 691-696.

Francq, C. and J-M. Zakoïan (2009) Bartlett's formula for a general class of non linear processes. Journal of Time Series Analysis, 30, 449-465. 